\title{
Interest Rate Term Structure Decomposition at the Instrument Level
}

\author{
Brian Barnard \\ Correspondence: Brian Barnard, Wits Business School, University of the Witwatersrand (WITS), South Africa.
}

Received: November 14, 2016

doi:10.11114/aef.v6i3.1975

\author{
Accepted: March 11, 2019 \\ Available online: April 4, 2019 \\ URL: https://doi.org/10.11114/aef.v6i3.1975
}

\begin{abstract}
The paper examines term structure decomposition at the instrument level - decomposing term structures for issues as well as the portfolio. Three different implementations are stipulated: axiomatic structural approaches, a sequential approach, and a base structure approach. The three different implementations are evaluated against a portfolio of risk-free government bonds. The goodness-of-fit and smoothness properties of instrument-level term structure decomposition are also considered. The conclusion points to remaining gaps in theory regarding instrument-level term structure decomposition, and considers areas of application - typically bond valuation.
\end{abstract}

Keywords: term structure decomposition, instrument valuation, bond valuation

\section{Introduction}

Barnard (2019) introduces and reviews term structure decomposition, its purpose and objective, the applicable models used to decompose term structures, and offers a brief comparison of these - their performance, strengths and weaknesses. Barnard (2019) also introduces an axiomatic structural approach as term structure decomposition method. In addition, the premises of term structure decomposition at the instrument level are also introduced. Term structure decomposition at the istrument level is then also the focus of this study. Next, the theoretical model thereof is stated.

\subsection{The Theoretical Model of Term Structure Decomposition at the Instrument Level}

Barnard (2019) notes that instrument-level term structure decomposition implies that a term structure is decomposed for each issue or instrument. Furthermore, hypothetically, the portfolio term structure is not directly decomposed, but derived from the issue term structures instead. Its aim is to offer a more direct way to simultaneously capture both the more predominantly unsystematic risk structures of issues, and the more predominantly systematic risk structure of the portfolio, through the mathematical relationship that links the two sets. It allows individual issue term structures to naturally deviate from the portfolio term structure, as it accommodates the unsystematic risk components of issues. This should advance the understanding of factors of pricing, in that it permits an analysis and comparison between individual issue term structures and the portfolio term structure, in line with the inquiry by Elton et al (2001), just in a more detailed and elaborate manner.

Issue term structures and the corresponding portfolio term structure are naturally and logically linked. Decomposing issue term structures implies that, for each issue, an issue-unique term structure of discount factors is devised, and discounting the issue's coupons at the issue-unique discount factors should equal the issue price. In addition, discounting the portfolio coupons at the portfolio term structure discount factors should equal the portfolio price. Overall, two relationships are preserved: First, the portfolio price obtained by discounting the portfolio coupons against the portfolio term structure, must equal the sum of the prices of the $\mathrm{N}$ issues associated with the portfolio, obtained by discounting each issue's coupons against each issue's term structure (equation 2.b). Secondly, and perhaps more importantly, discounting any portfolio coupon at the portfolio discount factor for a specific portfolio coupon date, must equal the sum of the corresponding issue coupons discounted at their specific issue discount factors for that coupon date (equation 1.a). Thus, the portfolio coupon $\left(C^{p}\right)$ for portfolio coupon date $\mathrm{k}$, discounted at the portfolio discount factor $\left(D F_{k}^{p}\right)$ of date $\mathrm{k}$, should equal the sum of the issue coupons $\left(C_{k}^{i}\right)$ of date $\mathrm{k}$, discounted at the corresponding issue discount factor $\left(D F_{k}^{i}\right)$ of date $\mathrm{k}$. For illustration, the portfolio coupon date, $\mathrm{k}$, an issue coupon and coupon date $I_{m}^{i}$ maps to, is notated as $P_{a[i][m]}$. 


$$
\begin{gathered}
D F_{k}^{p} C_{k}^{p}=\sum_{i}^{N}\left(D F_{k}^{i} C_{k}^{i}\right) ; k \in[1, \ldots, K] \\
I_{m}^{i} \rightarrow P_{a[i][m]}
\end{gathered}
$$

The above is captured by the optimization problem equation set 2. Given $\mathrm{N}$ issues, each with $M^{i}$ coupon dates, the difference between modelled issue prices $P_{n}^{i}$, and market issue prices $P_{n}^{i m}$ is minimized (equation 2.a). An equality constraint may be added to constrain the portfolio price to its actual value (equation 2.b), although it is not always mandatory. Inequality constraints are added to prevent negative issue (equation 2.c), and portfolio (equation 2.d) forward rates. The initial solution of equation 8 of Barnard (2019) would also function in this case. Here, issue value is predominantly derived from discounting issue coupons against issue discount factors $\left(D F_{k}^{i}\right)$, not portfolio discount factors $\left(D F_{k}^{p}\right)$, and the optimization primarily optimizes issue discount factors, not portfolio discount factors per se. Barnard (2019) discusses term structure decomposition as as optimization problem, and it is also further covered subsequently.

$$
\operatorname{minimize} \sum_{n=1}^{N}\left(P_{n}^{i m}-P_{n}^{i}\right)^{2}
$$

Subject to:

$$
\begin{gathered}
P^{p}=\sum_{k=1}^{K} D F_{k}^{p} C_{k}^{p}=\sum_{i=1}^{N} \sum_{m=1}^{M^{i}} D F_{m}^{i} C_{m}^{i} \\
D F_{1}^{p} \leq 1 ; D F_{k}^{p} \geq D F_{(k+1)}^{p} ; D F_{K}^{p} \geq 0 ; k \in[2, \ldots, K-1] \\
D F_{1}^{i} \leq 1 ; D F_{m}^{i} \geq D F_{(m+1)}^{i} ; D F_{M^{i}}^{i} \geq 0 ; i \in[1, \ldots, N] ; m \in\left[2, \ldots, M^{i}-1\right]
\end{gathered}
$$

The problem with the optimization problem of equation set 2 is that, although it defines the relationship between issue and portfolio term structures, and although it permits issue and portfolio term structures to be decomposed, the resultant structures would generally have poor smoothness, as little is stipulated in this regard.

According to Marciniak (2006), term structure models are characterized by: 1) goodness of fit (flexibility), 2) smoothness, 3) stability of results (robustness to changes in the data), and 4) numerical stability and time of computation. Stability in turn relates to four different properties: 1) robustness to outliers, 2) robustness to minor changes in the data set, 3) (non)uniqueness of estimates, and 4) numerical stability. Both Marciniak (2006) and Bolder and Gusba (2002) point to the trade-off between flexibility (goodness of fit), smoothness and stability. Excess flexibility leads to loss of smoothness (humps) and to a drastic fall in its stability. It is expected that the criteria of term structure decomposition should naturally extend to the case of decomposing term structures at the instrument level. Also, it is an open question whether the trade-off between flexibility and smoothness would be dominant in the case of instrument level term structure decomposition as well.

\subsection{Axiomatic Structural Approaches}

In order to introduce structure smoothness to equation set 2, the axiomatic structural approaches of Barnard (2019) may be considered. Barnard (2019) notes that the premise that the resultant portfolio term structure optimizes goodness of fit against structure smoothness, may already stipulate sufficient information to decompose a term structure, without necessarily stipulating a set of basis functions or splines, as conventional models do. Rather, it may simply be stated as a general optimization problem, by including constraints to guide the structure or smoothness thereof (Boyd and Vandenberghe, 2004). He outlines the optimization problem, and shows how the model is applied to a portfolio of bond instruments. The model agrees with conventional models in terms of its primary objective to minimize issue price modelling error. However, instead of basing constraints on the acceptable output structure, on mathematical functions, it is rather based on the attributes of the structure. Two attributes were considered: the structure has minimum variance, 
and the growth rate between any two points likely do not exceed a given value.

With regards to inducing optimal structure, Bolder and Gusba (2002) state that zero-coupon curve smoothness is a relevant criterion for a term-structure estimation model, because overly non-smooth zero-coupon curves are highly oscillatory functions in a model. This implies the occurrence of dramatic swings in rates from one period to the next. Typically, the term structure of interest rates is expected to move gradually across the term-to-maturity spectrum. Dramatic moves, conversely, are not considered reasonable. There is not really a possible economic reason to explain a large difference between the price of a five-year pure discount bond and a five-year-and-one-week pure discount bond.

Marciniak (2006) notes that parsimonious models infer several basic shapes: monotonous increasing or decreasing, humped, or S-shaped. The value of one of the coefficients should correspond to zero-coupon rates for ultra long maturities. At the short end of the curve, the sum of some of the parameter values should be equal to the level of the shortest interest rates. Bolder et al (2004) in turn note the Merrill Lynch exponential spline (MLES) model is strictly based on curve-fitting techniques. That is, it is a strictly mathematical process defined as fitting a continuous function to a set of discretely observed data points. The process of generating the yield curve makes no underlying economic assumptions, nor does it impose any functional form to the yield curve. It models the discount function as a linear combination of exponential basis functions.

In light of this, Barnard (2019) took the primary constraint on the output structure as the requirement that individual rates must grow gradually, with neighbouring or periods of rates not demonstrating dramatic moves. In addition, the short end of the curve may be constrained to conform with immediate term expectations. This may be accomplished by assigning weights to issues and their price residuals, according to their maturities (Bolder and Gusba, 2002; Marciniak, 2006).

Therefore, for purposes of conventional term structure decomposition, to constrain variance in the output structure, Barnard (2019) added another constraint to the optimization problem: the output variance $\left(V^{p}\right)$ may not exceed a certain ceiling $\left(V^{p C}\right)$, defined in advance (equation 3.a). Output variance is based on the portfolio forward rates $\left(F^{p}\right)$, and is measured as the forward rate growth rates. It can also be argued that another property of the term structure is that the growth rate between any two points would never exceed a certain rate $\left(V^{p p C}\right)$ (equation 3.b).

$$
\begin{gathered}
V^{p}=\sum_{k=1}^{K-1}\left(\frac{F_{t_{k}^{k+1}}^{p}-F_{t_{k-1}^{k}}^{p}}{t_{k}^{k+1}}\right)^{2} \leq V^{p C} \\
=\sum_{k=1}^{K-1}\left(-1 / t_{k}^{k+1} \cdot 1 / t_{k}^{k+1} \ln \left(D F_{k+1}^{p} / D F_{k}^{p}\right)+1 / t_{k}^{k+1} \cdot 1 / t_{k-1}^{k} \ln \left(D F_{k}^{p} / D F_{k-1}^{p}\right)\right)^{2} \\
=\sum_{k=1}^{K-1}\left(-a_{k} \cdot \ln \left(D F_{k+1}^{p}\right)+\left(a_{k}+b_{k}\right) \cdot \ln \left(D F_{k}^{p}\right)-b_{k} \cdot \ln \left(D F_{k-1}^{p}\right)\right)^{2} \\
\left(\frac{F_{t_{k}^{k+1}}^{p}-F_{t_{k-1}^{k}}^{p}}{t_{k}^{k+1}}\right)^{2} \leq V^{p p c} ; k \in[1, \ldots, K-1]
\end{gathered}
$$

Thus, in the case of instrument level term structure decomposition, in order to constrain the smoothness of the issue and portfolio term structures, a number of potential structure axioms are noted. A basic degree of smoothness is expected over individual issues' term structures, and this should naturally extend to the portfolio term structure. In addition, it is expected that issues should have discount factors and forward rates comparable to that of the portfolio for comparable dates. Given that they relate to similar periods in time, and even though their risk profiles differ, the absolute distance between issue and portfolio discount factors, is expected to be minimum. As an extension, because the interval lengths of issues and the portfolio differ, issues' discount factors may be expected to be comparable to all portfolio discount factors falling within issues' coupon intervals. Similarly, issues are expected to have comparable discount factors and forward rates over their overlapping coupon intervals, even though their risk profiles also differ. Though different issue intervals do not perfectly overlap, a number of issue intervals do overlap. Also, because forward rates ought to be more sensitive, absolute distance constraints can be referenced in terms of forward rates, rather than discount factors.

Below, equation set 4 to 7 build on the axioms stated above. All the equation sets are extensions of the basic optimization problem of equation set 2. In these equations, $C^{i v}, C^{i p d}, C^{i p f d}, C^{i i f d}$ and $C^{p v}$ are all different ceiling or maximum values.

Equation set 4 shows an optimization problem that seeks to define structure through minimum issue structure variance 
(equation 4.e), and minimum issue to portfolio discount factor absolute distance (equation 4.f), for each and every issue coupon date. In the equation, $N$ refers to the number of issues, and $M^{i}$ refers to the coupon dates of each issue $i$. $F_{t_{m+1}^{m}}^{i}$ is the forward rate of an issue $i$ over its coupon interval $t_{m}^{m+1} . D F_{m}^{i}$ is the discount factor of issue $i$ for the coupon date $m . D F_{a[i][m]}^{p}$ is the portfolio discount factor corresponding to the coupon date $m$ of issue $i$. Three variants of the problem are possible: minimizing issue structure variance only, minimizing issue-portfolio discount factor absolute distance, and minimizing both.

$$
\begin{gathered}
\operatorname{minimize} \sum_{n=1}^{N}\left(P_{n}^{i m}-P_{n}^{i}\right)^{2} \\
\text { subject to: } \\
P^{p}=\sum_{k=1}^{K} D F_{k}^{p} C_{k}^{p}=\sum_{i=1}^{N} \sum_{m=1}^{M^{i}} D F_{m}^{i} C_{m}^{i} \\
D F_{1}^{p} \leq 1 ; D F_{k}^{p} \geq D F_{(k+1)}^{p} ; D F_{K}^{p} \geq 0 ; k \in[2, \ldots, K-1] \\
\sum_{i=1}^{N} \sum_{m=1}^{M^{i}-1}\left(\frac{\left.F_{t_{m}^{m+1}}^{i}-F_{t_{m-1}^{m}}^{i}\right)^{2} \leq C^{i v}}{t_{m}^{m+1}}{ }^{i} \geq D F_{(m+1)}^{i} ; D F_{M^{i}}^{i} \geq 0 ; i \in[1, \ldots, N] ; m \in\left[2, \ldots, M^{i}-1\right]\right. \\
\sum_{i=1}^{N} \sum_{m=1}^{M^{i}-1}\left(-1 / t_{m}^{m+1} \cdot 1 / t_{m}^{m+1} \ln \left(D F_{m+1}^{i} / D F_{m}^{i}\right)+1 / t_{m}^{m+1} \cdot 1 / t_{m-1}^{m} \ln \left(D F_{m}^{i} / D F_{m-1}^{i}\right)\right)^{2} \leq C^{i v} \\
\sum_{i=1}^{N} \sum_{m=1}^{M^{i}-1}\left(-a_{m} \cdot \ln \left(D F_{m+1}^{i}\right)+\left(a_{m}+b_{m}\right) \cdot \ln \left(D F_{m}^{i}\right)-b_{m} \cdot \ln \left(D F_{m-1}^{i}\right)\right)^{2} \leq C^{i v} \\
M_{m}^{i}\left(D F_{a[i][m]}^{p}\right)^{2} \leq C^{i p d}
\end{gathered}
$$

Equation set 5 shows an optimization problem that seeks to define structure through minimum issue structure variance (equation 5.e), and minimum issue to portfolio forward rate absolute distances (equation 5.f). The distances are measured per issue interval, and against all portfolio intervals that overlap with a particular issue interval. $F_{m}^{i}$ is the forward rate of an issue $i$ over its coupon interval demarcated by the coupon dates $m$ and $m-1 . D F_{a[i][m][j]}^{p}$ and $F_{a[i][m][j]}^{p}$ are respectively the portfolio discount factor and forward rate of a portfolio interval falling within the issue interval demarcated by the coupon date $m$ and $m-1$ of issue $i . t_{j}^{j+1}$ refers to the portfolio interval, and $J^{i m}$ is the number of portfolio intervals falling within a particular issue interval demarcated by the coupon date $m$ and $m-1$ of issue $i$.

$$
\begin{gathered}
\operatorname{minimize} \sum_{n=1}^{N}\left(P_{n}^{i m}-P_{n}^{i}\right)^{2} \\
\text { subject to: } \\
P^{p}=\sum_{k=1}^{K} D F_{k}^{p} C_{k}^{p}=\sum_{i=1}^{N} \sum_{m=1}^{M^{i}} D F_{m}^{i} C_{m}^{i}
\end{gathered}
$$




$$
\begin{gathered}
D F_{1}^{p} \leq 1 ; D F_{k}^{p} \geq D F_{(k+1)}^{p} ; D F_{K}^{p} \geq 0 ; k \in[2, \ldots, K-1] \\
D F_{1}^{i} \leq 1 ; D F_{m}^{i} \geq D F_{(m+1)}^{i} ; D F_{M^{i}}^{i} \geq 0 ; i \in[1, \ldots, N] ; m \in\left[2, \ldots, M^{i}-1\right] \\
\sum_{i=1}^{N} \sum_{m=1}^{M^{i}-1}\left(\frac{F_{t_{m}^{m+1}}^{i}-F_{t_{m-1}^{m}}^{i}}{t_{m}^{m+1}}\right)^{2} \leq C^{i v} \\
\sum_{i=1}^{N} \sum_{m=1}^{M^{i}-1}\left(-1 / t_{m}^{m+1} \cdot 1 / t_{m}^{m+1} \ln \left(D F_{m+1}^{i} / D F_{m}^{i}\right)+1 / t_{m}^{m+1} \cdot 1 / t_{m-1}^{m} \ln \left(D F_{m}^{i} / D F_{m-1}^{i}\right)\right)^{2} \leq C^{i v} \\
\sum_{i=1}^{i v} \sum_{m=1}^{N} \sum_{j=1}^{M^{i}-1}\left(-a_{m} \cdot \ln \left(D F_{m+1}^{i}\right)+\left(a_{m}+b_{m}\right) \cdot \ln \left(D F_{m}^{i}\right)-b_{m} \cdot \ln \left(D F_{m-1}^{i}\right)\right)^{2} \leq C^{i v} \\
\left.\sum_{i=1}^{N} \sum_{m=1}^{M^{i}} \sum_{j=1}^{J^{i m}}\left(\ln \left(D F_{m}^{i}\right)-\ln \left(D F_{m-1}^{i}\right) F_{m}^{i}-F_{a[i][m][j]}^{p}\right)^{2} \leq C^{i p f d}\left(\ln \left(D F_{a[i][m][j]}^{p}\right)-\ln \left(D F_{a[i][m][j-1]}^{p}\right)\right)\right)^{2} \leq C^{i p f d}
\end{gathered}
$$

Equation set 6 shows an optimization problem that seeks to define structure through minimum issue structure variance (equation 6.e), and minimum issue to issue forward rate absolute distances (equation 6.f). The distances are measured per issue interval, and the issues against which the distances are measured may be either ordinary issues or reference issues with issue intervals that overlap with a particular issue interval. Reference intervals of reference issues are further discussed in a subsequent section. $F_{m}^{i}$ is the forward rate of an issue $i$ over its coupon interval demarcated by coupon date $m$ and $m-1 . F_{a[i][m][j]}^{i}$ is the forward rate of an ordinary or reference issue overlapping with the issue interval of issue $i$ demarcated by its coupon date $m$ and $m-1 . t_{l}^{l+1}$ refers to the overlapping ordinary or reference issue interval, and $L^{i m}$ is the number of overlapping issue intervals falling within a particular issue interval demarcated by the coupon date $m$ and $m-1$ of issue $i$.

$$
\begin{gathered}
\operatorname{minimize} \sum_{n=1}^{N}\left(P_{n}^{i m}-P_{n}^{i}\right)^{2} \\
\text { subject to: } \\
P^{p}=\sum_{k=1}^{K} D F_{k}^{p} C_{k}^{p}=\sum_{i=1}^{N} \sum_{m=1}^{M^{i}} D F_{m}^{i} C_{m}^{i} \\
D F_{1}^{p} \leq 1 ; D F_{k}^{p} \geq D F_{(k+1)}^{p} ; D F_{K}^{p} \geq 0 ; k \in[2, \ldots, K-1] \\
D F_{m}^{i} \geq D F_{(m+1)}^{i} D F_{M^{i}}^{i} \geq 0 ; i \in[1, \ldots, N] ; m \in\left[2, \ldots, M^{i}-1\right] \\
\sum_{i=1}^{N} \sum_{m=1}^{M^{i}-1}\left(\frac{F_{t_{m}^{m+1}}^{i}-F_{t_{m-1}^{m}}^{i}}{t_{m}^{m+1}}\right)^{2} \leq C^{i v}
\end{gathered}
$$




$$
\begin{gathered}
\sum_{i=1}^{N} \sum_{m=1}^{M^{i}-1}\left(-1 / t_{m}^{m+1} \cdot 1 / t_{m}^{m+1} \ln \left(D F_{m+1}^{i} / D F_{m}^{i}\right)+1 / t_{m}^{m+1} \cdot 1 / t_{m-1}^{m} \ln \left(D F_{m}^{i} / D F_{m-1}^{i}\right)\right)^{2} \leq C^{i v} \\
\sum_{i=1}^{N} \sum_{m=1}^{M^{i}-1}\left(-a_{m} \cdot \ln \left(D F_{m+1}^{i}\right)+\left(a_{m}+b_{m}\right) \cdot \ln \left(D F_{m}^{i}\right)-b_{m} \cdot \ln \left(D F_{m-1}^{i}\right)\right)^{2} \leq C^{i v} \\
\qquad \sum_{i=1}^{N} \sum_{m=1}^{M^{i}} \sum_{l=1}^{L^{i m}}\left(F_{m}^{i}-F_{a[i][m][l]}^{i}\right)^{2} \leq C^{i i f d} \\
\sum_{i=1}^{N} \sum_{m=1}^{M^{i}} \sum_{l=1}^{L^{i m}}\left(-t_{m-1}^{m}\left(\ln \left(D F_{m}^{i}\right)-\ln \left(D F_{m-1}^{i}\right)\right)+t_{l-1}^{l}\left(\ln \left(D F_{a[i][m][l]}^{i}\right)-\ln \left(D F_{a[i][m][l-1]}^{i}\right)\right)\right)^{2} \leq C^{i p f d}
\end{gathered}
$$

Equation set 7 shows an optimization problem that seeks to define structure through minimum issue structure variance (equation 7.e), and minimum portfolio structure variance (equation 7.f). $F_{t_{k}^{k+1}}^{p}$ refers to the portfolio forward rate of portfolio interval $t_{k}^{k+1}$.

$$
\begin{aligned}
& \operatorname{minimize} \sum_{n=1}^{N}\left(P_{n}^{i m}-P_{n}^{i}\right)^{2} \\
& \text { subject to: } \\
& P^{p}=\sum_{k=1}^{K} D F_{k}^{p} C_{k}^{p}=\sum_{i=1}^{N} \sum_{m=1}^{M^{i}} D F_{m}^{i} C_{m}^{i} \\
& D F_{1}^{p} \leq 1 ; D F_{k}^{p} \geq D F_{(k+1)}^{p} ; D F_{K}^{p} \geq 0 ; k \in[2, \ldots, K-1] \\
& D F_{1}^{i} \leq 1 ; D F_{m}^{i} \geq D F_{(m+1)}^{i} ; D F_{M^{i}}^{i} \geq 0 ; i \in[1, \ldots, N] ; m \in\left[2, \ldots, M^{i}-1\right] \\
& \sum_{i=1}^{N} \sum_{m=1}^{M^{i}-1}\left(\frac{F_{t_{m}^{m+1}}^{i}-F_{t_{m-1}^{m}}^{i}}{t_{m}^{m+1}}\right)^{2} \leq C^{i v} \\
& \sum_{i=1}^{N} \sum_{m=1}^{M^{i}-1}\left(-1 / t_{m}^{m+1} \cdot 1 / t_{m}^{m+1} \ln \left(D F_{m+1}^{i} / D F_{m}^{i}\right)+1 / t_{m}^{m+1} \cdot 1 / t_{m-1}^{m} \ln \left(D F_{m}^{i} / D F_{m-1}^{i}\right)\right)^{2} \leq C^{i v} \\
& \sum_{i=1}^{N} \sum_{m=1}^{M^{i}-1}\left(-a_{m} \cdot \ln \left(D F_{m+1}^{i}\right)+\left(a_{m}+b_{m}\right) \cdot \ln \left(D F_{m}^{i}\right)-b_{m} \cdot \ln \left(D F_{m-1}^{i}\right)\right)^{2} \leq C^{i v} \\
& \sum_{k=1}^{K-1}\left(\frac{F_{t_{k}^{k+1}}^{p}-F_{t_{k-1}^{k}}^{p}}{t_{k}^{k+1}}\right)^{2} \leq C^{p v}
\end{aligned}
$$




$$
\begin{gathered}
\sum_{k=1}^{K-1}\left(-1 / t_{k}^{k+1} \cdot 1 / t_{k}^{k+1} \ln \left(D F_{k+1}^{p} / D F_{k}^{p}\right)+1 / t_{k}^{k+1} \cdot 1 / t_{k-1}^{k} \ln \left(D F_{k}^{p} / D F_{k-1}^{p}\right)\right)^{2} \leq C^{p v} \\
\sum_{k=1}^{K-1}\left(-a_{k} \cdot \ln \left(D F_{k+1}^{p}\right)+\left(a_{k}+b_{k}\right) \cdot \ln \left(D F_{k}^{p}\right)-b_{k} \cdot \ln \left(D F_{k-1}^{p}\right)\right)^{2} \leq C^{p v}
\end{gathered}
$$

\subsection{Sequential Approach}

A sequential approach builds on the premise that certain issues predominate as reflection of accurate value and thus risk, over certain periods of their lifetime. One instance may be liquid issues generally seen as reference bonds. Another interpretation may be that, over a given interval, the issue to mature next generally has the greatest impact on portfolio structure formation (as part of conventional term structure decomposition) over that interval, and is likely the most accurate reflection of risk for that particular interval. Under this view, an issue does not necessarily form a reference over its entire lifetime, but only over a limited interval. From this it is not difficult to see how different issues can serve as reference issues over different issue and portfolio intervals.

A term structure is then sequentially constructed by requiring issues to reference the structure of applicable reference issues over applicable reference intervals, and letting issues attain their individual value with an unique structure over their remaining, tail intervals. Each issue has a number of intervals that overlap with reference intervals, and can thus reference these, or even adopt the structure of these. Also, each issue has a number of tail intervals that are not referenced against reference intervals, predominantly because the issue predominates over its tail interval. Should the resultant structure that attain issue value over the tail interval contain too much variance, the issue structure over the preceding intervals - the intervals referenced to reference intervals - can be adjusted accordingly.

An advantage of the sequential approach is its simplicity, compared to the optimization problems of the axiomatic structural approach. Even though they do not always yield practical results, their offer simple solutions.

\subsection{Base-Structure Approach}

A drawback of the sequential approach is that, the structure devised over the tail interval of an issue may impact subsequent issues' structures, and overall structure smoothness, when the particular issue serves as reference issue over some of its tail intervals. This is predominantly due to its sequential nature. Instead, when devising the structure over the tail intervals of issues, subsequent issues and overall structure smoothness should equally be considered essentially a concurrent, non-sequential approach. In light of this, the base structure approach devises issue structures from a reference structure that is concurrently calculated, and that considers overall structure smoothness. For this purpose, a standard portfolio term structure decomposed via conventional means serves as the perfect candidate.

The base structure approach essentially allows two variants, that either favour issue structure smoothness, or portfolio structure smoothness.

As the first variant, issue term structures are devised by having each issue adopt the base structure, and subsequently shifting issue structures away from the base structure, to attain their individual value. Issue risk relative to the portfolio should both be interval-generic, and interval-specific. Issue risk over its entire term should be generic in the sense that, relative to the portfolio, the issue maintains a certain risk profile. At the same time, interval specific risk deviations can also occur - even though issue risk over its intervals is general, specific deviations may also occur over particular intervals.

Shifting issue term structures impact the derived portfolio term structure. As part of this approach, the derived portfolio term structure may differ from the base structure, and may contain excess variance. In its pure form, this approach essentially disregards negative portfolio forward rates, and the corresponding portfolio term structure. It thus disregards the second premise of the relationship between issue and portfolio term structures, and only regards the first premise portfolio value indeed matches the sum of issue value, but the relationship between issue and portfolio discount factors are not strictly adhered to. The optimization of equation set 8 may be run to constrain negative portfolio forward rates and portfolio structure variance (equation 8.a), whilst maintaining issue value (equation 8.b). The portfolio forward rates of equation 8.a are rewritten in terms of portfolio discount factors, which in turn are rewritten in terms of issue discount factors.

To implement this variant, the forward rates of issues are shifted, and the rates are uniformly shifted. This aligns with a generic view of issue risk, relative to the portfolio. Equally, this should represent the fact that issue risk generally accumulate over intervals. 
As the second variant, the base case is sustained such that it essentially forms the derived portfolio term structure as well. This approach adheres to both premises of the relationship between issue and portfolio term structures. Equation set 9 captures an optimization that seeks to minimize issue value, when preserving the base case. $D F_{k}^{b}$ refers to the portfolio discount factor of the base structure for the portfolio coupon date $\mathrm{k}$.

$$
\begin{gathered}
\operatorname{minimize} \sum_{k=1}^{K-1}\left(\frac{F_{t_{k}^{k+1}}^{p}-F_{t_{k-1}^{k}}^{p}}{t_{k}^{k+1}}\right)^{2} \\
=\sum_{k=1}^{K-1}\left(-a_{k} \cdot \ln \left(D F_{k+1}^{p}\right)+\left(a_{k}+b_{k}\right) \cdot \ln \left(D F_{k}^{p}\right)-b_{k} \cdot \ln \left(D F_{k-1}^{p}\right)\right)^{2} \\
=\sum_{k=1}^{K-1}\left(-a_{k} \cdot \ln \left(\sum_{n}^{N_{k+1}} D F_{n}^{i} C_{n}^{i} / C_{k+1}^{p}\right)+\left(a_{k}+b_{k}\right) \ln \left(\sum_{n}^{N_{k}} D F_{n}^{i} C_{n}^{i} / C_{k}^{p}\right)-b_{k} \cdot \ln \left(\sum_{n}^{N_{k-1}} D F_{n}^{i} C_{n}^{i} / C_{k-1}^{p}\right)\right)^{2} \\
\operatorname{subject~to:}_{D F_{1}^{i} \leq 1 ; D F_{m}^{i} \geq D F_{(m+1)}^{i} ; D F_{M^{i}}^{i} \geq 0 ; i \in[1, \ldots, N] ; m \in\left[2, \ldots, M^{i}-1\right]}^{M_{m}^{i} C_{m}^{i} ; i \in[1, \ldots, N]} \\
\text { minimize } \sum_{n=1}^{N}\left(P_{n}^{i m}-P_{n}^{i}\right)^{2}
\end{gathered}
$$

Subject to:

$$
\begin{gathered}
D F_{k}^{b}=D F_{k}^{p}=\sum_{n}^{N^{k}} D F_{n}^{i} C_{n}^{i} ; k \in[1, \ldots, K-1] \\
D F_{1}^{p} \leq 1 ; D F_{k}^{p} \geq D F_{(k+1)}^{p} ; D F_{K}^{p} \geq 0 ; k \in[2, \ldots, K-1] \\
D F_{1}^{i} \leq 1 ; D F_{m}^{i} \geq D F_{(m+1)}^{i} ; D F_{M^{i}}^{i} \geq 0 ; i \in[1, \ldots, N] ; m \in\left[2, \ldots, M^{i}-1\right]
\end{gathered}
$$

\section{Methodology}

To test the implementations, a market term structure was simulated by selecting a number of intervals, randomly selecting the forward rates of the interval points between a set minimum (0) and maximum (0.3), and interpolating the forward rates of the remaining points by means of straight lines across the intervals. Such a structure may perhaps not be practical. Nevertheless, it poses a difficult term structure to decompose, and may help to illustrate model accuracy.

All government zero coupon and vanilla bonds from the South African market for a given date (2014-07-09) were taken. Issue term structures were sourced for these bonds by equating the issue term structures to the simulated portfolio term structure, to preserve the relationship between the issue and portfolio term structure. The government bonds were discounted against the simulated issue term structures, to obtain simulated values for these issues, using their original coupon data.

The issues' simulated prices and original coupon data were used to decompose term structures, and the results were compared to the simulated structures. The given sample constitutes 27 issues, with 211 portfolio coupon dates. Some of the implementations were also run on the original price data of the said government bond sample.

To implement the sequential approach, a custom algorithm was written. To source a base structure for the base structure approach, the decomposition model of Barnard (2019) was used.

\section{Analysis}

Overall, the optimization problems of the axiomatic, structural approach yield poor results, from a structural smoothness perspective. Although they attain low or zero issue value residuals, they struggle to define proper structure. Yet, they highlight key points. The sequential approach yields an average result, and, on face-value, even surpasses the output of the optimization problems, from a structural perspective, regardless of its drawbacks. The base structure approach yields satisfactory results, that attain both goodness of fit and structure.

Figure 1 and 2 show the simulated issue and portfolio term structures. Figure 1 depicts only the issue term structures, and figure 2 depicts the issue and portfolio term structures. 
Figure 3 to 17 pertain to the optimization problems of the axiomatic structural approach. Figure 3 to 8 correspond to variants of equation set 4 . In the first case (figure 3 and 4), only issue structure variance is constrained. In the second case (figure 5 and 6), only issue to portfolio discount factor absolute distance is constrained. In the third case, issue structure variance, and issue to portfolio discount factor absolute distance, are jointly constrained. In all cases, the ceilings are set at values that permit zero issue value residuals. Also, in also cases, the issue term structures carry the broad or general structure of the simulated structures, to some extent.

With regards to the first case, issue structure variance is primarily an issue structure constraint, and can only indirectly influence the portfolio structure, through the relationship that holds between the issue and portfolio structures. What is noteworthy is that constrained issue variance does not naturally extend to the portfolio structure. Therefore, constraining issue structure variance may not necessarily imply that portfolio structure variance is constrained, such that it is no longer necessary to constrain it as well.

Moving to the second case, even though the issue to portfolio discount factor absolute distances are significantly constrained (lower than 1e-03), it does not adequately shape or define structure - some similitude is expected between the issue and portfolio structures, and too little of that is seen. The conclusion is simply that similar or identical issue and portfolio discount factors do not guarantee similar or identical issue and portfolio forward rates. Also, similar or identical issue and portfolio discount factors do not guarantee forward rate consistency - particularly that of the portfolio - as is required for basic structure. In most cases, the portfolio has additional, intermediary intervals, due to other issue intervals (imperfectly) overlapping with an issue interval. There must be general consistency across the portfolio discount factors (or forward rates) falling within an issue interval, for the issue and portfolio forward rates to compare.

As figure 7 shows, the two different constraints do complement each other, but not nearly as sufficient to devise proper structure.

In all the cases, the portfolio non-negative forward rate constraint prevents negative portfolio forward rates. In all the above-mentioned cases, the need to perhaps additionally constrain portfolio forward rate consistency or variance, is pointed out.

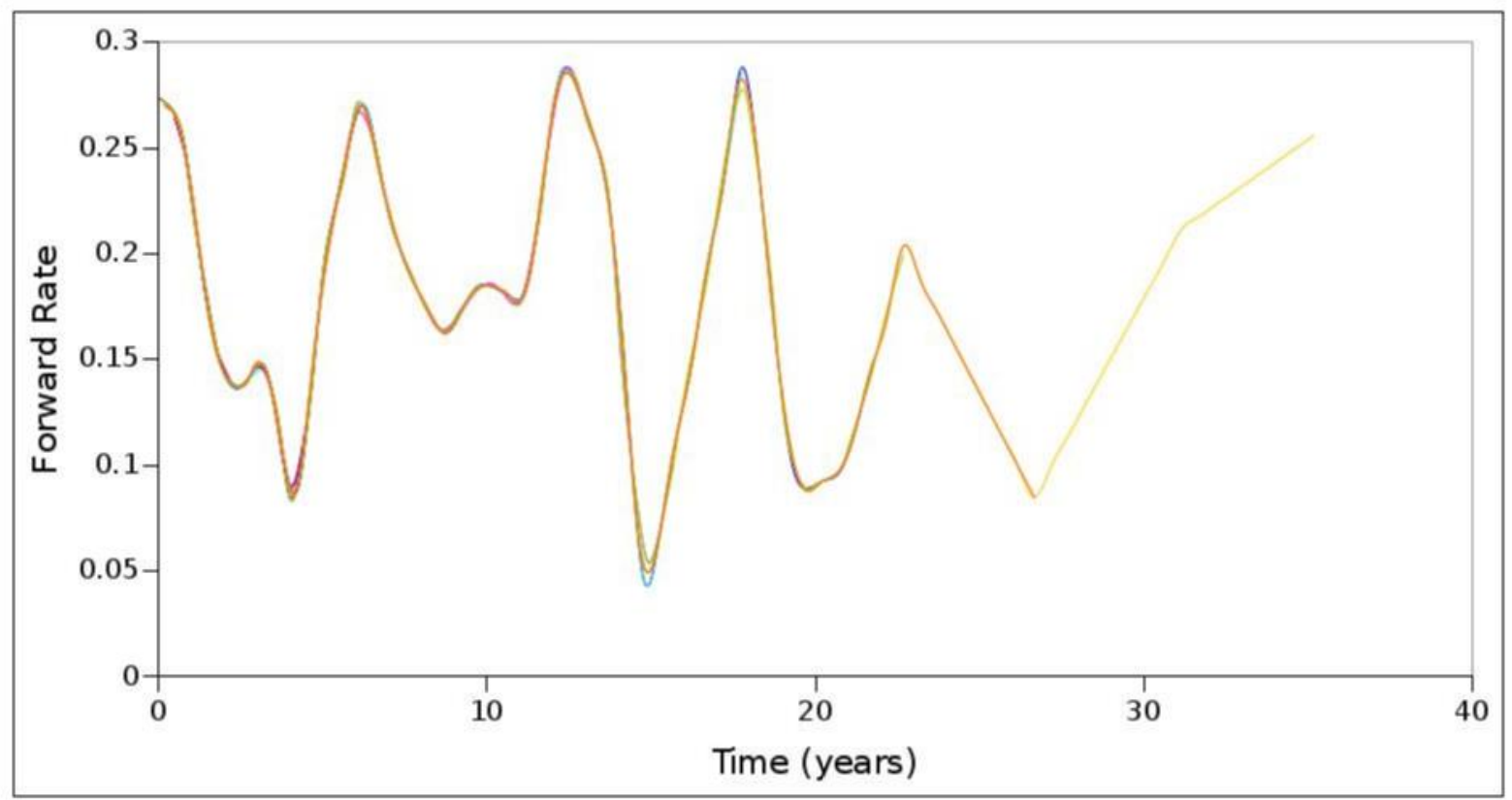

Figure 1. Simulated issue term structures 


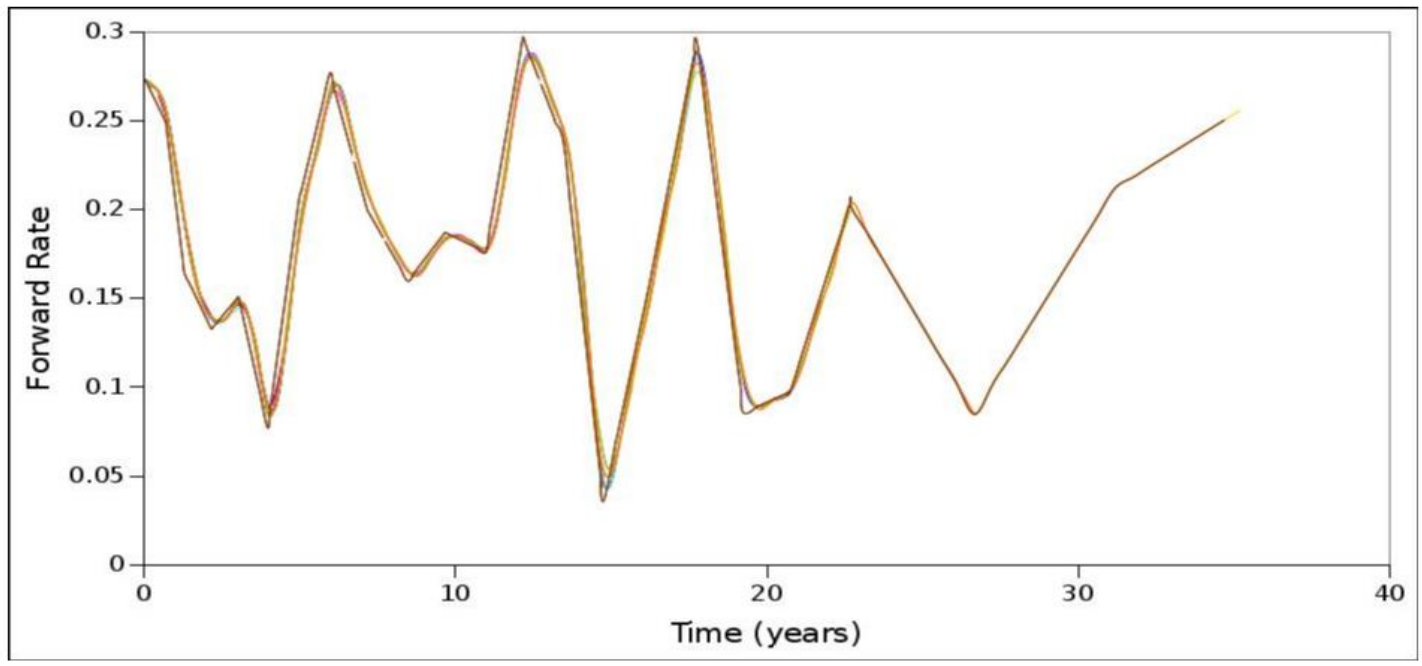

Figure 2. Simulated issue and portfolio term structures

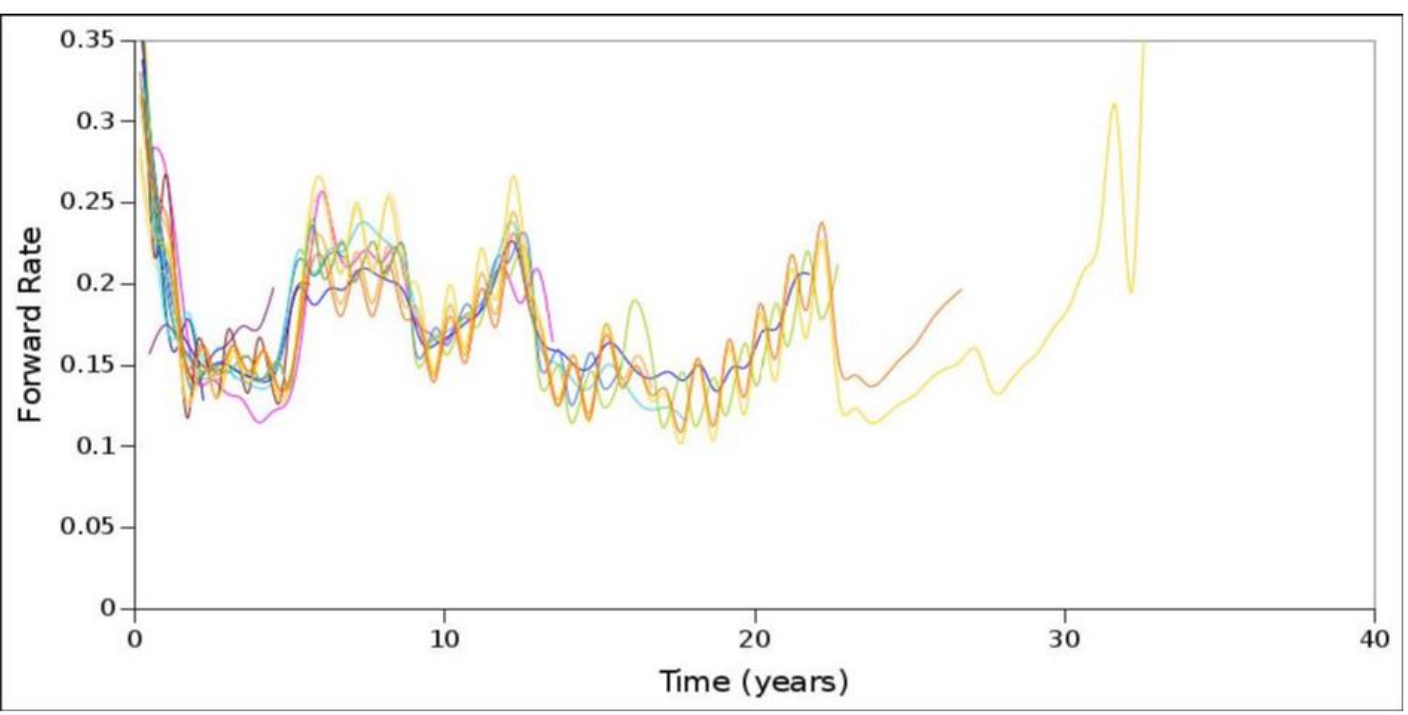

Figure 3. Modelled issue term structures - issue structure variance constrained

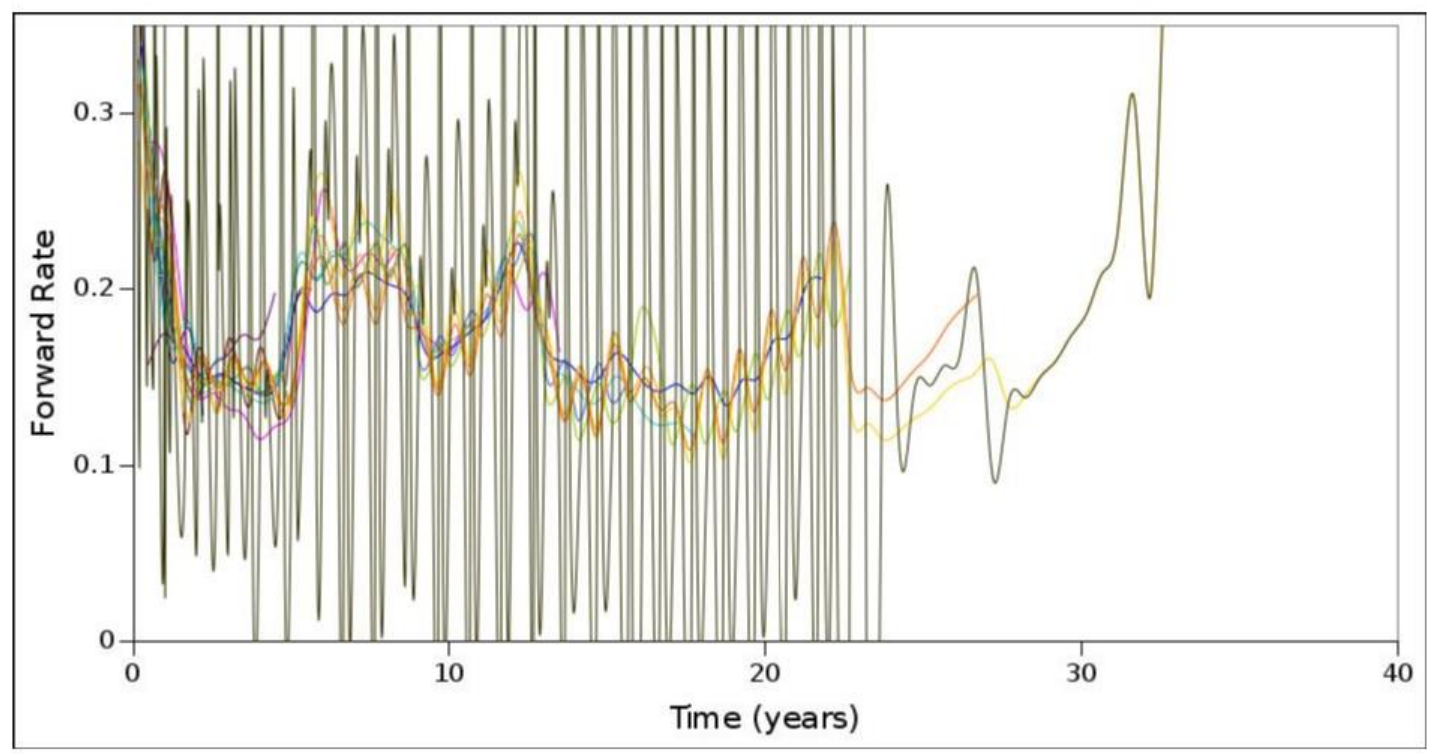

Figure 4. Modelled issue and portfolio term structures - issue structure variance constrained 


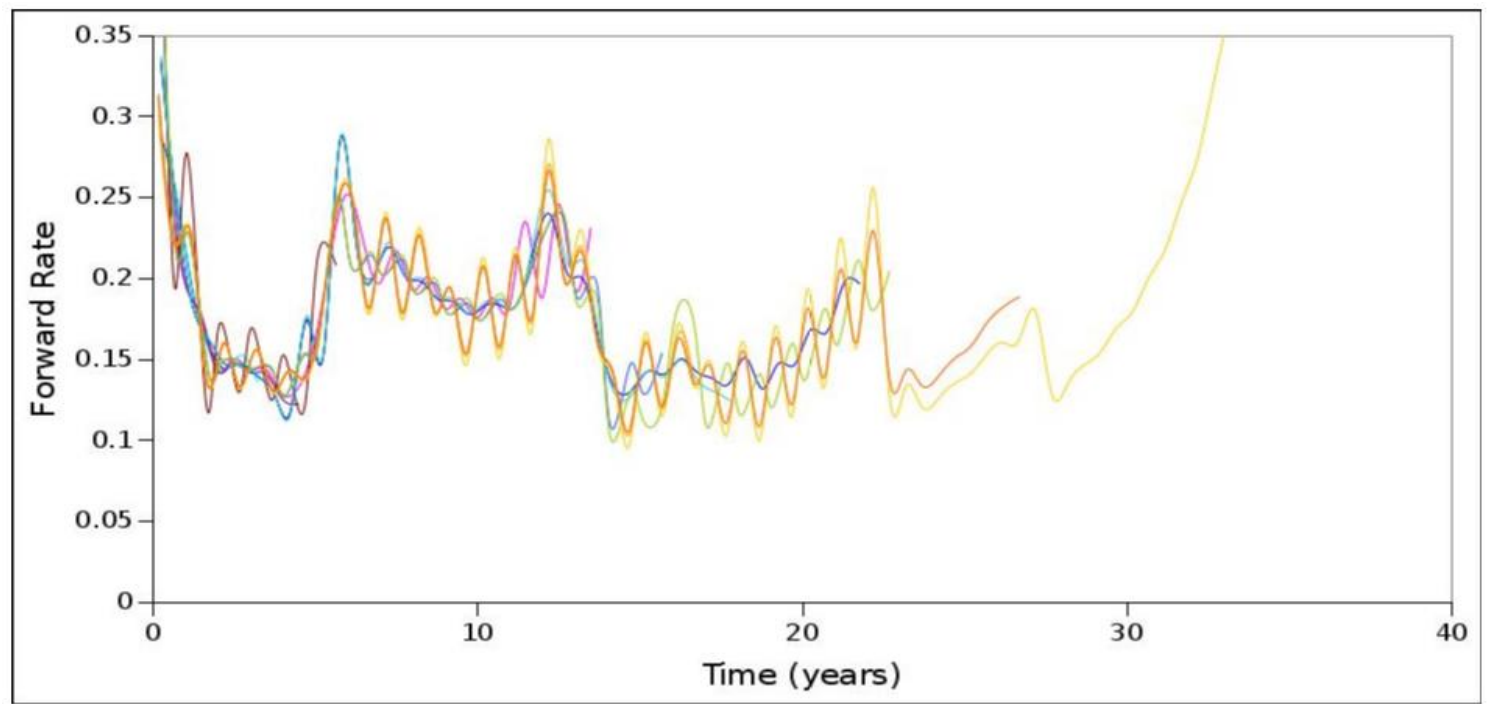

Figure 5. Modelled issue term structures - issue to portfolio discount factor distance constrained

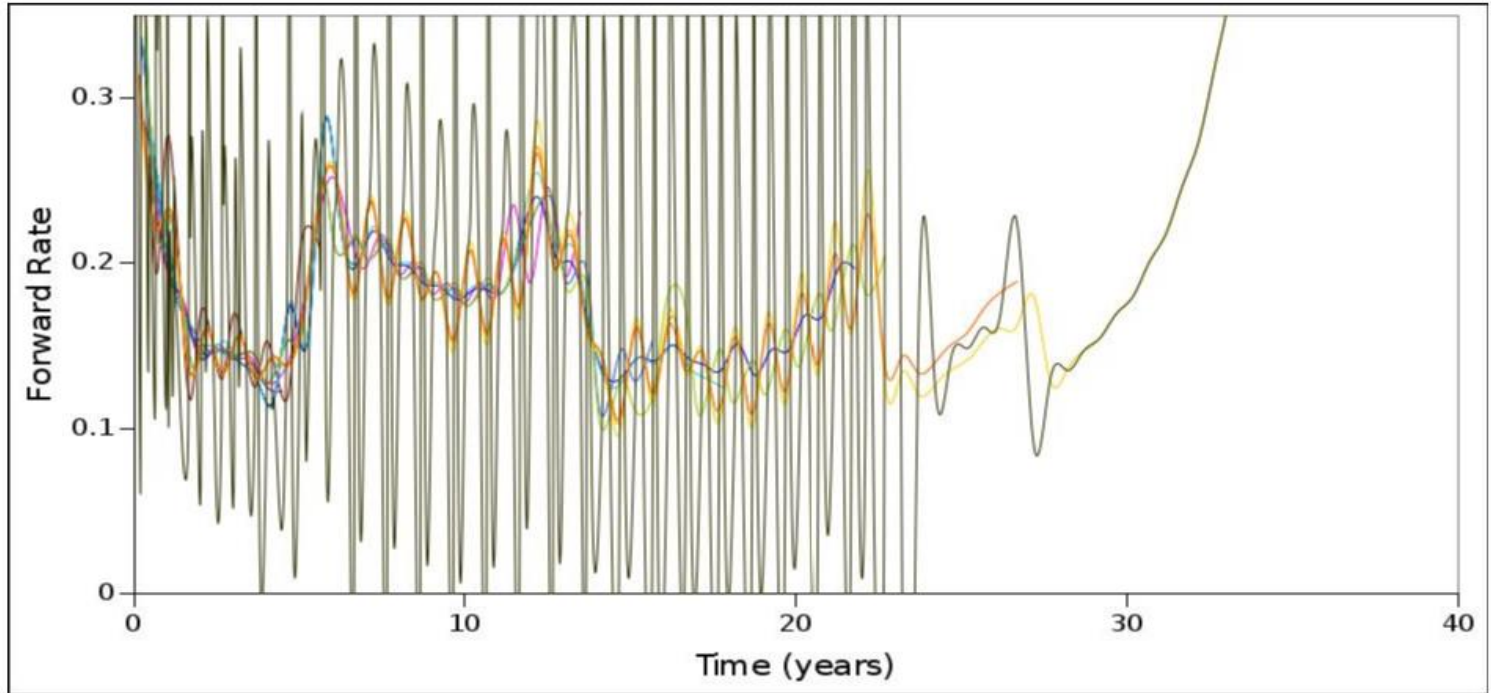

Figure 6. Modelled issue and portfolio term structures - issue to portfolio discount factor distance constrained

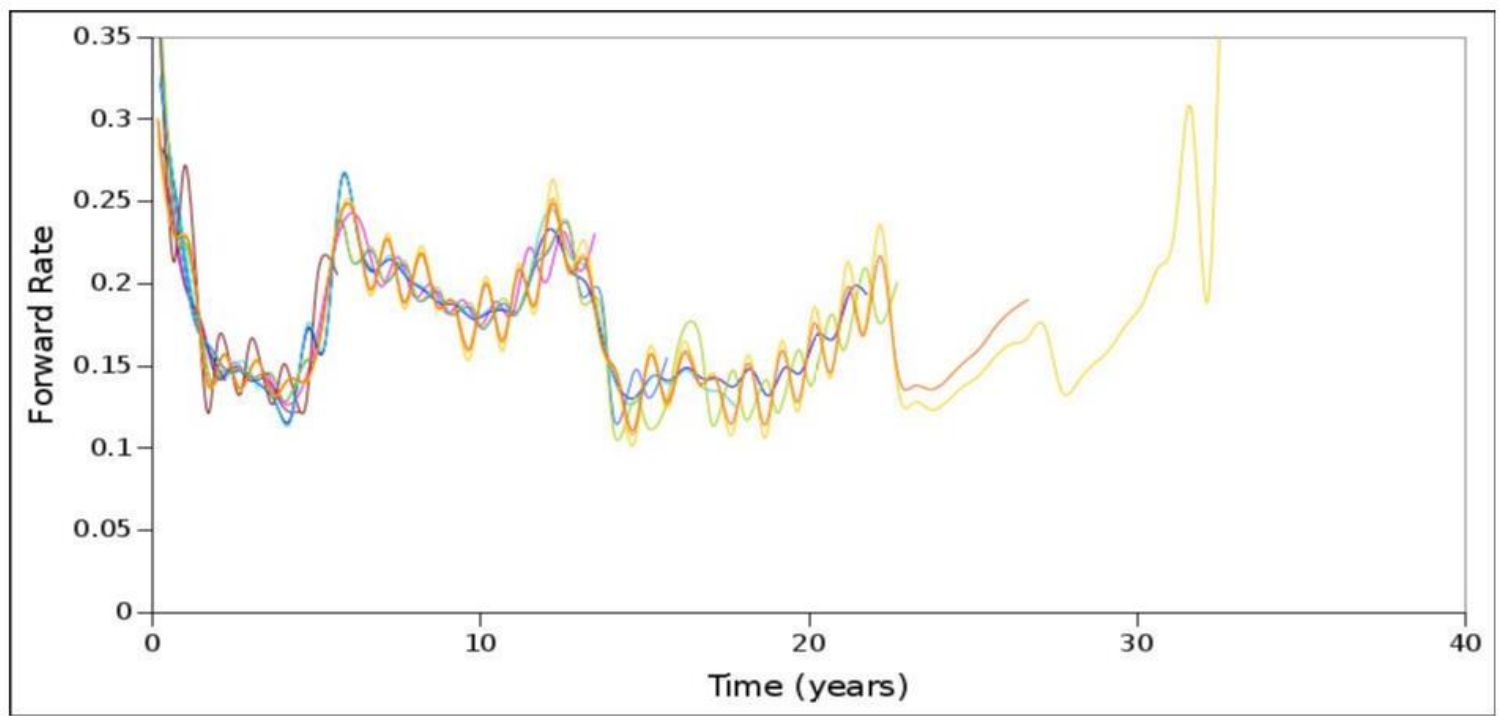

Figure 7. Modelled issue term structures - issue structure variance, and issue to portfolio discount factor distance constrained 


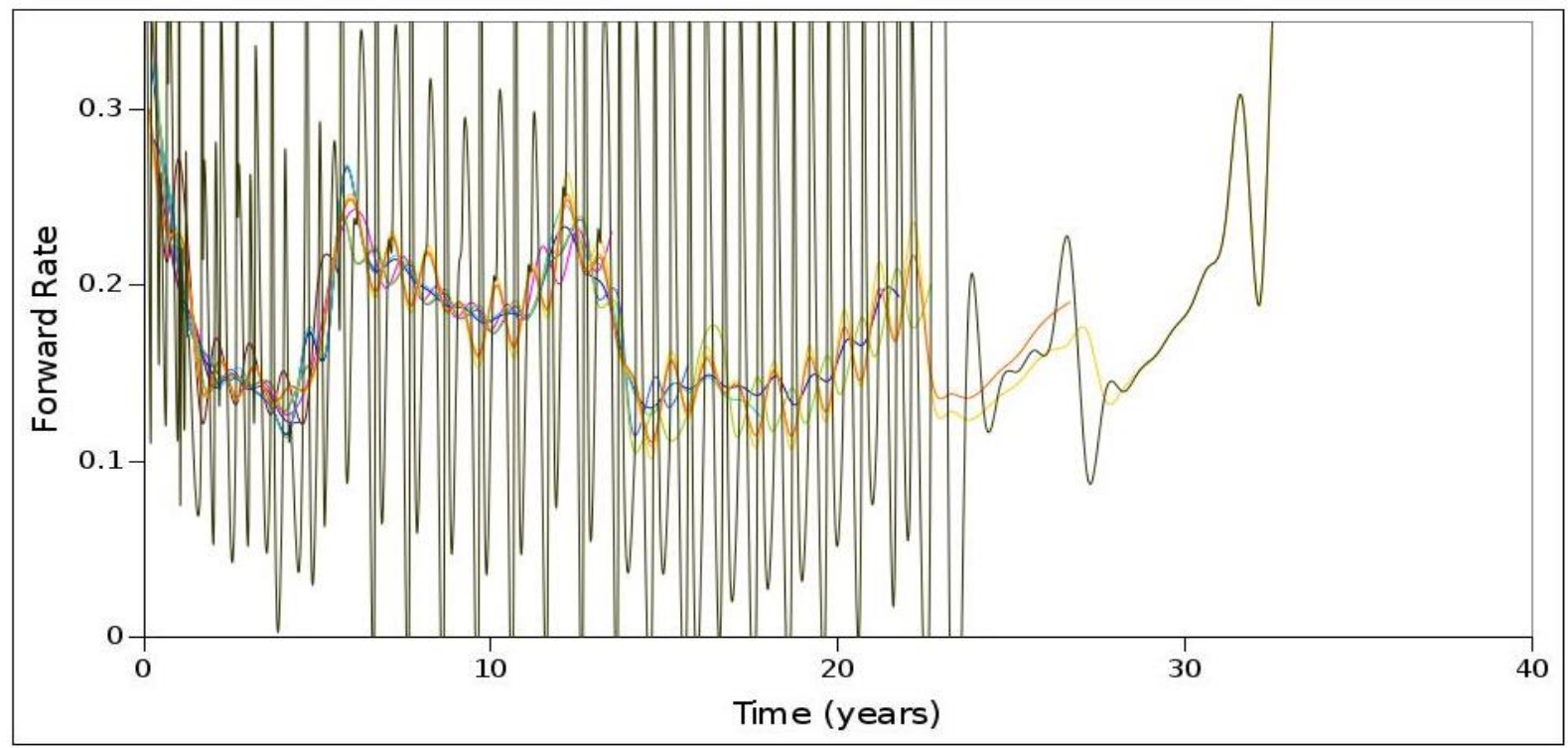

Figure 8. Modelled issue and portfolio term structures - issue structure variance, and issue to portfolio discount factor distance constrained

Figure 9 to 12 pertain to equation set 5. In the first case, issue to portfolio forward rate absolute distance is constrained for all portfolio intervals overlapping with a particular issue interval. Again, the ceiling is set at the level that permits zero issue value residuals. Even though the premise of the constraint is a likely characteristic of a well-defined structure, it seems insufficient to define structure on its own. Significant differences still exist between issue and portfolio forward rates, that can only be further reduced by jeopardizing achieved issue value residuals. This may point to having fallen behind a local optimum, which reiterate the need for an additional or stronger constraint to prevent or overcome this. Portfolio forward rate consistency is still lacking and it seems that the constraint adds little in this regard. This negatively reflects on the hypothesis that portfolio forward rate consistency naturally follows when issue to portfolio forward rate distances are optimal. In fact, it may suggest the converse: issue to portfolio forward rate distances are optimal when portfolio forward rate consistency is secured.

In this case, constraining issue structure variance as well (figure 11 and 12) significantly benefits the structure, perhaps even more than what can be ascribed to the issue structure variance constraint alone (figure 3 and 4). This seconds the proposition made previously: to adequately define structure, an additional or stronger constraint may be required, over and above constraining issue to portfolio forward rate absolute distance.

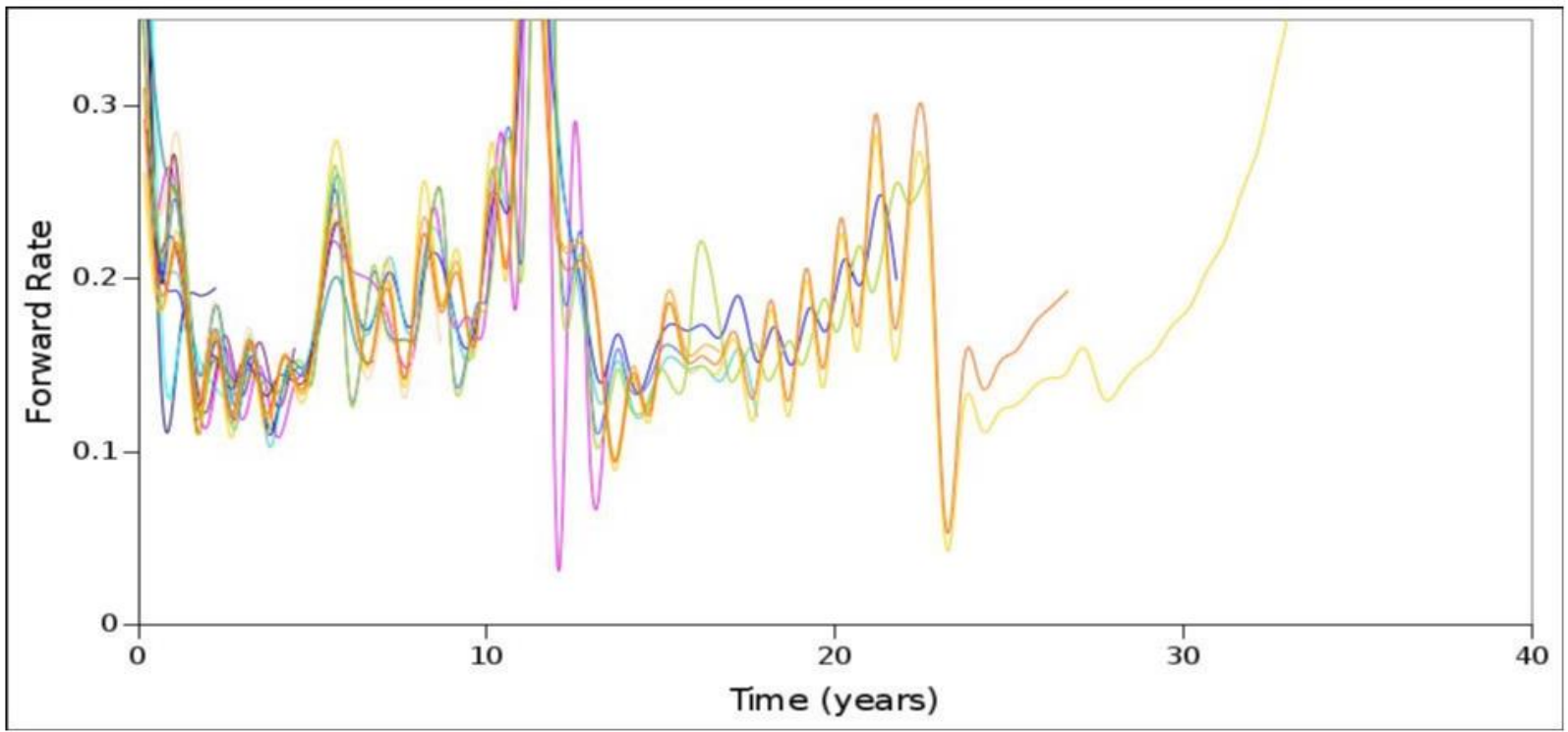

Figure 9. Modelled issue term structures - issue to portfolio forward rate absolute distance constrained 


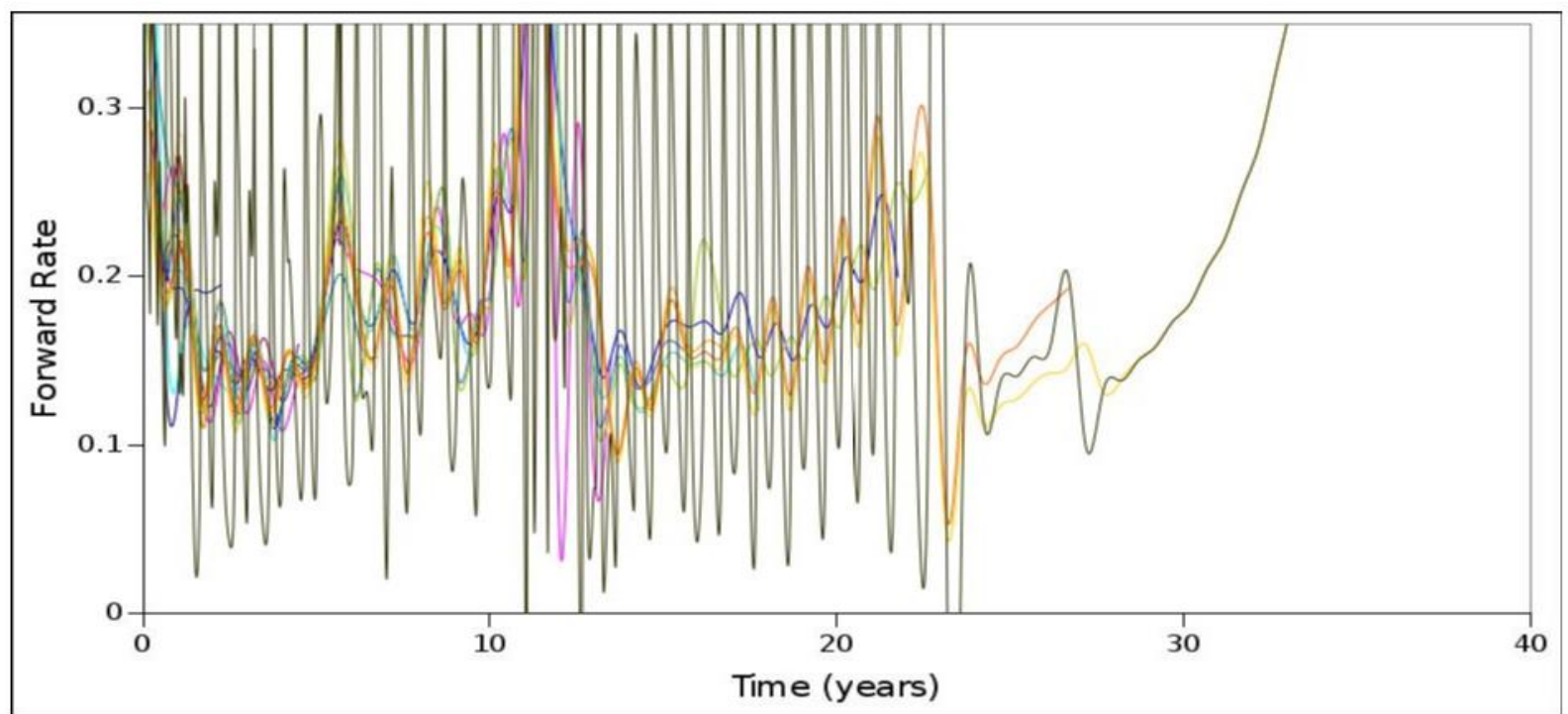

Figure 10. Modelled issue and portfolio term structures - issue to portfolio forward rate absolute distance constrained

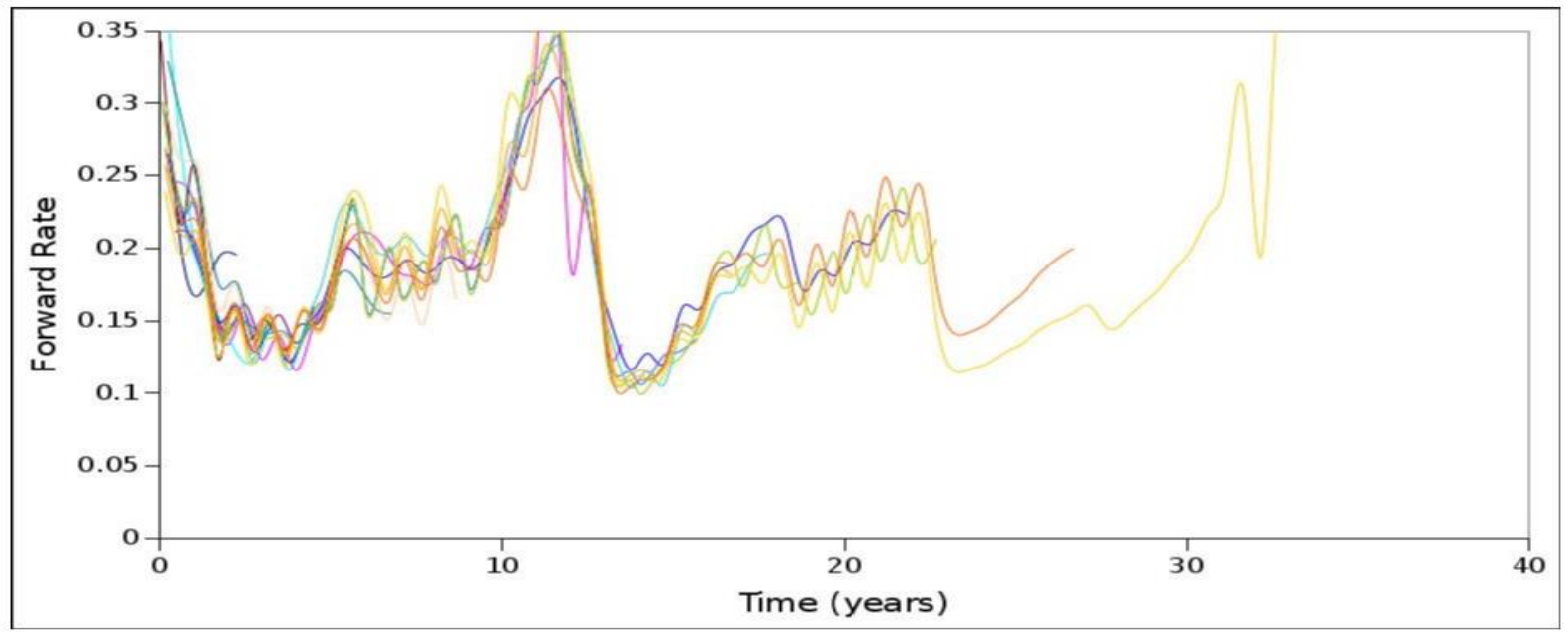

Figure 11. Modelled issue term structures - issue to portfolio forward rate absolute distance, and issue structure variance constrained

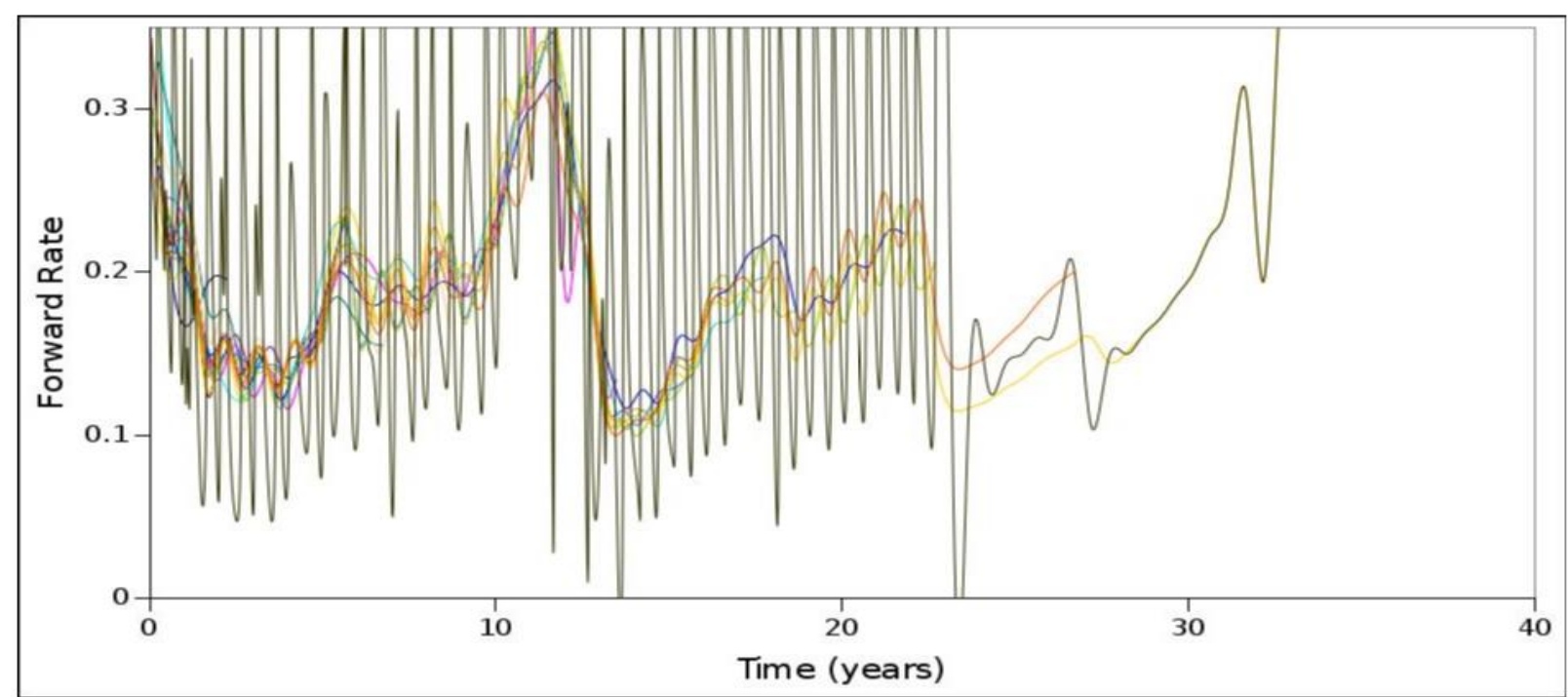

Figure 12. Modelled issue and portfolio term structures - issue to portfolio forward rate absolute distance, and issue structure variance constrained 
Figure 13 to 15 pertain to equation set 6 . Here, issue to reference issue forward rate absolute distances are constrained. Reference issues are selected in much the same way as that of the sequential approach. As the outcome of the sequential approach subsequently demonstrates, structure can be derived from reference issue sub-structures to a great extent. Portfolio negative forward rates are not constrained as part of the first variant, but indeed as part of the second variant. Ceiling values reflect the point at which issue value residuals are zero. Evidently, the forward rates of a number of issues follow each other - issues indeed follow reference issues. Further lowering the ceiling jeopardizes issue value residuals, but supports the point that issues do indeed align with reference issues. However, the outcome questions whether reference issues can be utilized in a non-sequential capacity. In a pure sequential setup, reference issues essentially carry precedence over their intervals. On the other hand, the optimization problem does not readily differentiate between reference issues and ordinary issues, when calculating distances or steps, nor does it assign preference.

The drastic change in structure when returning the portfolio negative forward rate constraint (figure 14), may reflect on the power contained within the portfolio forward rate to define structure.

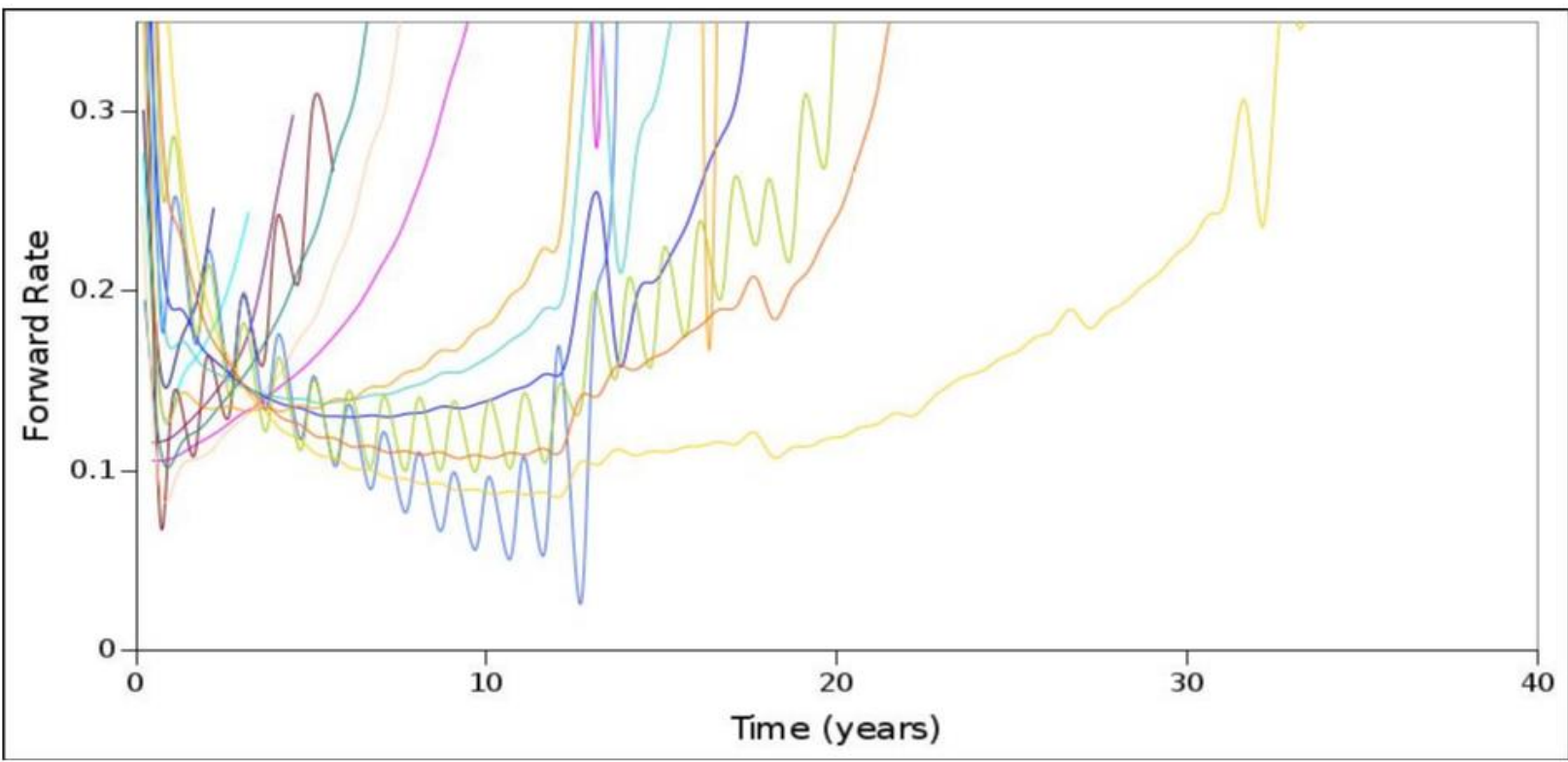

Figure 13. Modelled issue term structures - issue to reference issue forward rate absolute distance constrained, with portfolio negative forward rates unconstrained

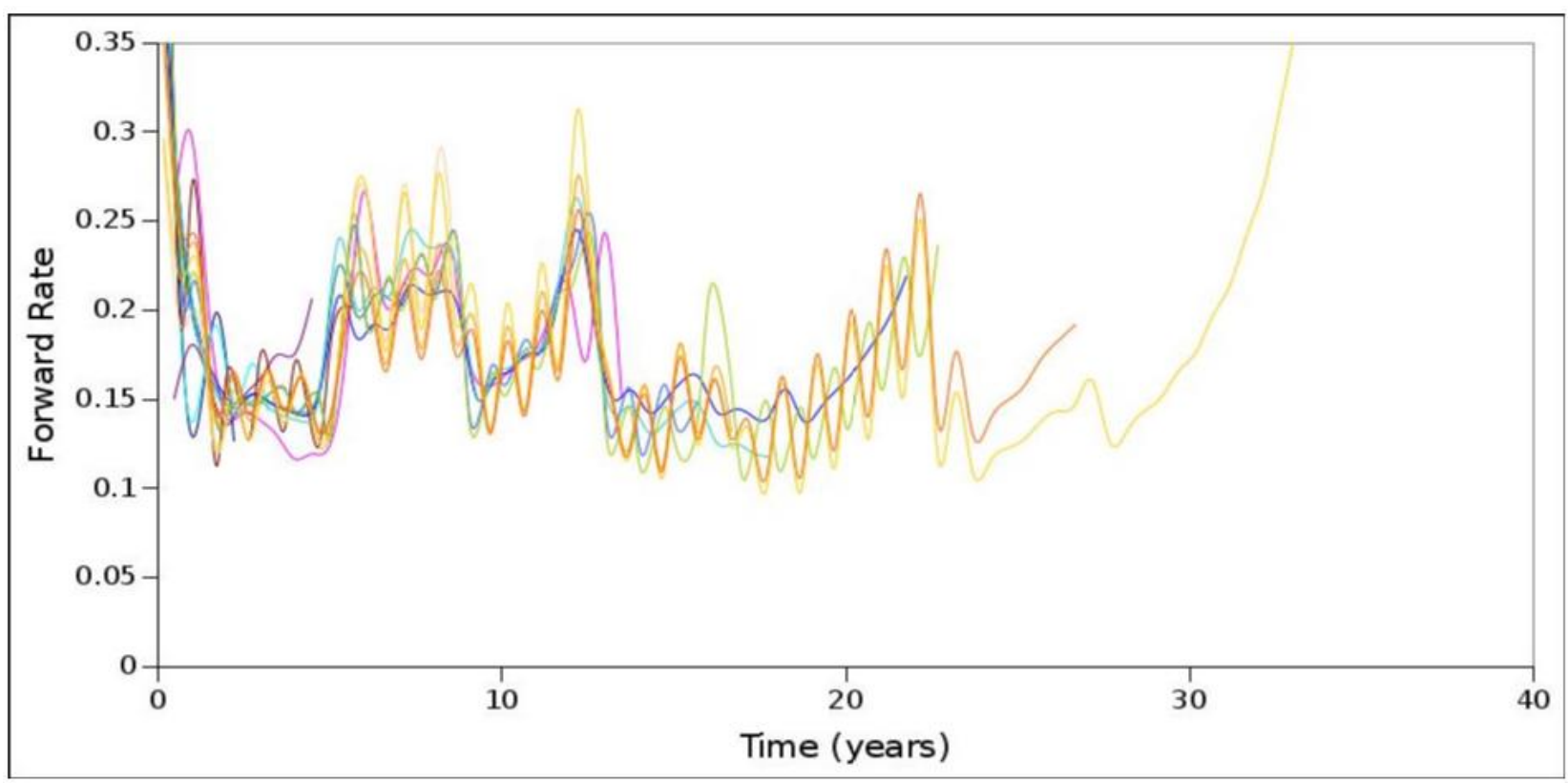

Figure 14: Modelled issue term structures - issue to reference issue forward rate absolute distance constrained 


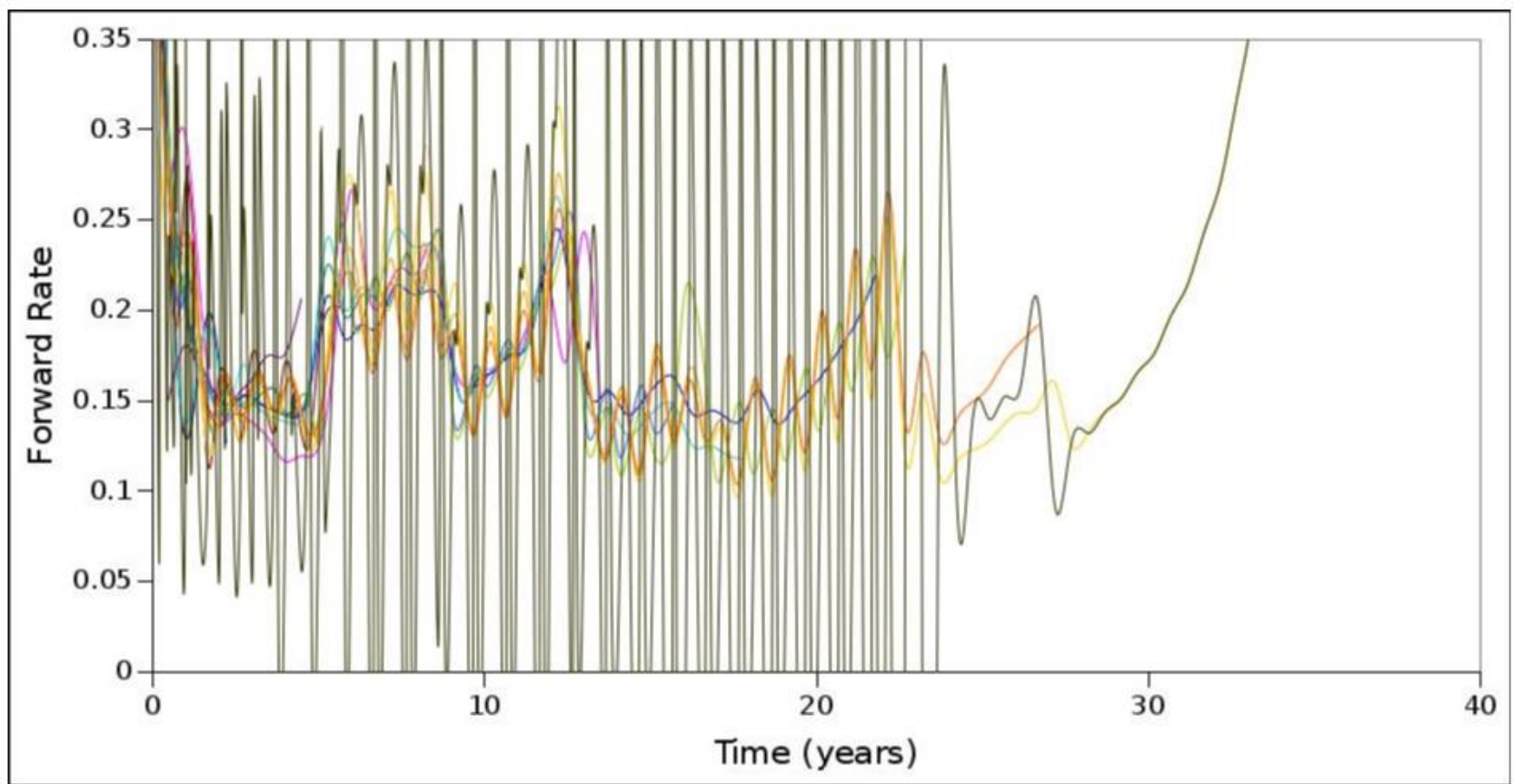

Figure 15. Modelled issue and portfolio term structures - issue to reference issue forward rate absolute distance constrained

Figure 16 and 17 pertain to equation set 7 . Here, the portfolio structure variance is constrained. Although the portfolio structure is by far smoother than previous variants, this variant struggles to attain zero issue value residuals, with significant residuals remaining (5\%). Related to this, the variant still struggles to devise a proper structure that resembles the simulated structure. The conclusion is that portfolio structure smoothness too is not sufficient to guarantee proper structure. Issue value through issue term structures are too distant and indirect to induce structure in the portfolio structure. In light of this, it is suggested that issue value - discounted against the derived portfolio term structure - is added as additional objective function, to help shape the portfolio structure. This would be in addition to issue value discounted against issue term structures as primary objective function (equation 2.a). This would be very similar to the base structure approach then.

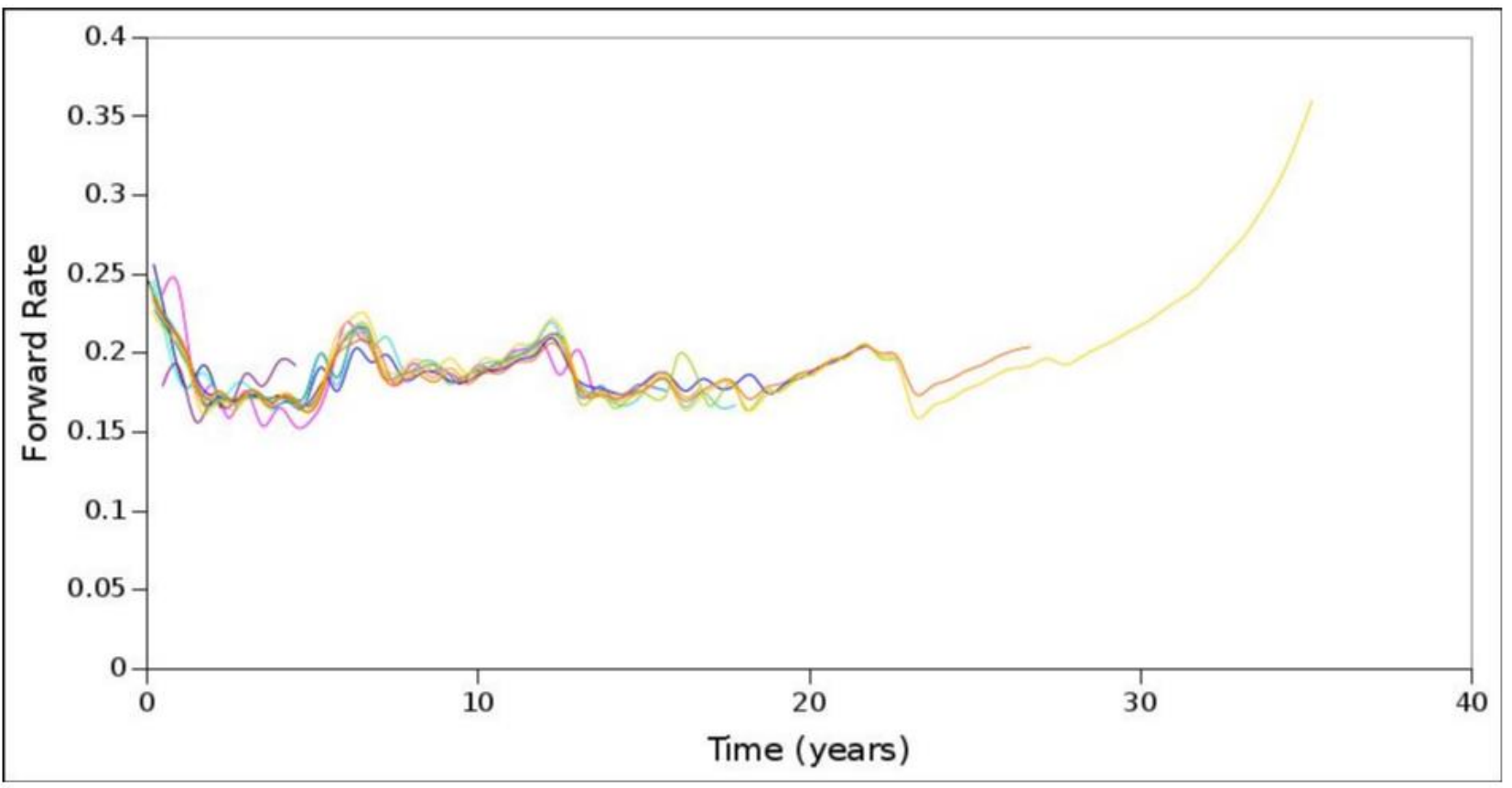

Figure 16. Modelled issue term structures - portfolio structure variance constrained 


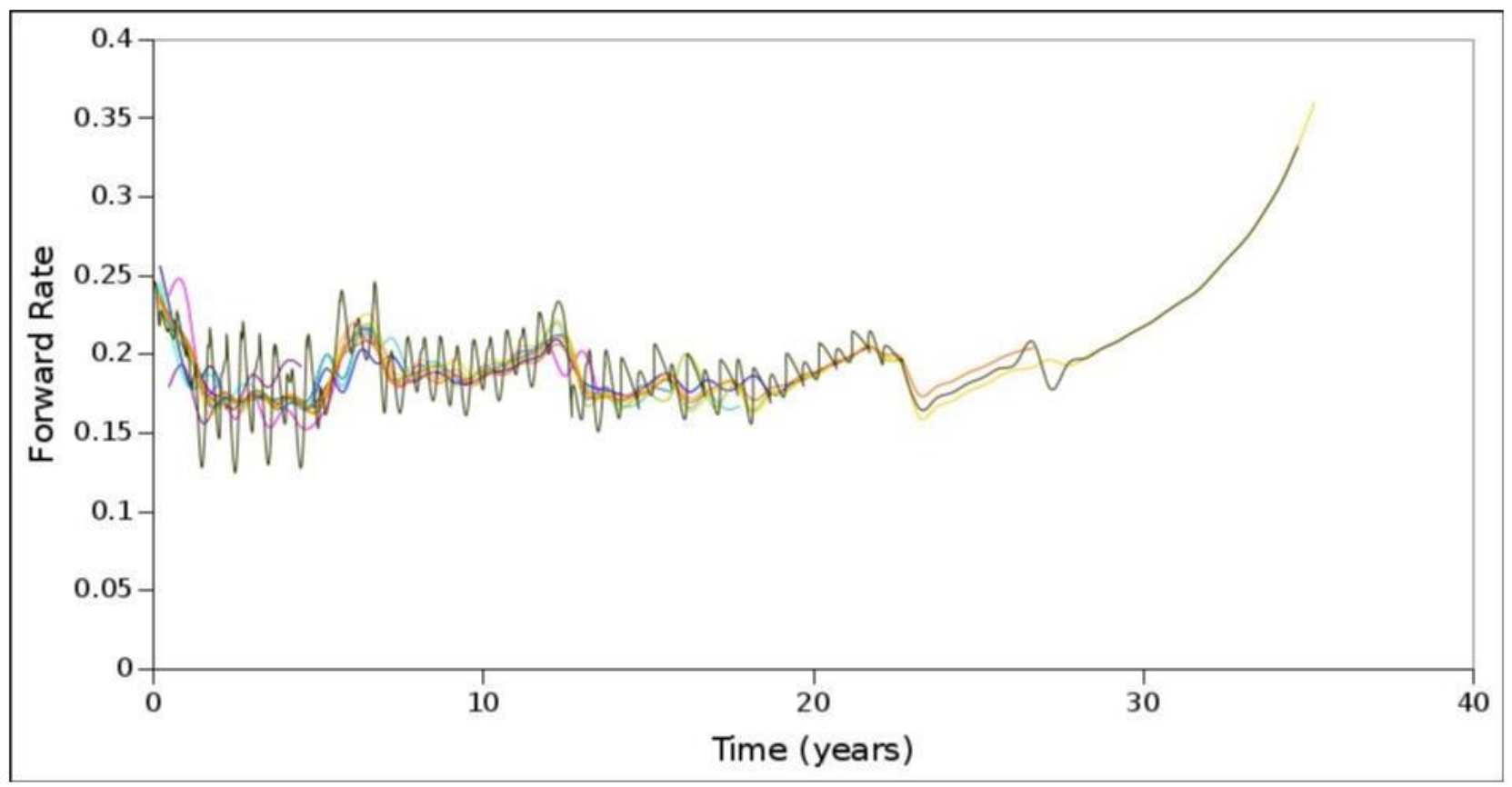

Figure 17. Modelled issue and portfolio term structures - portfolio structure variance constrained

Figure 18 depicts the sequential approach. It will not receive a lot of attention here, as it is surpassed by the base structure approach. However, its result shows there are grounds to its premise: predominant issues as reference issues do carry precedence over their applicable, reference intervals; structure can be significantly derived from predominant issues over their applicable reference intervals. It weakness is also affirmed - subsequent - and thus overall sub-structure smoothness is affected by preceding sub-structure smoothness.

Figure 19 to 28 pertain to the base structure approach. Figure 19 and 20 depict the results when the base structure approach is fed the simulated case. Because the simulated issue term structures were derived from the simulated portfolio term structure, there are no issue value residuals, and thus little challenge after sourcing the portfolio term structure as base structure, which the model is well able to do. Hence, figure 21 to 28 all pertain to the market case. Figure 21 and 22 utilize a base structure decomposed at a variance ceiling of 0.15 . Figure 23 to 28 utilize a base structure decomposed at a variance ceiling of 0.35 .

Figure 23 and 24 reflect on the first variant of the base structure approach. Issue term structures are devised by having each issue adopt the base structure, and subsequently shifting issue structures away from the base structure, to attain their individual value. The result is very smooth issue term structures that guarantee zero issue value residuals in all cases. This is generally at the cost of portfolio structure variance and consistency. Figure 25 and 26 show the outcome of the optimization that seeks to minimize portfolio forward rate variance, whilst maintaining issue value (equation set 8). Although portfolio forward rate variance is reduced, it is not altogether removed, and negative forward rates still occur. The result of this is a severe degradation in issue structure smoothness that is difficult to justify.

Figure 27 and 28 pertain to the second variant of the base structure approach - the base structure is maintained to form the eventual portfolio term structure as well. Figure 27 and 28 show the result of the optimization that seeks to minimize issue value, whilst preserving the base structure (equation set 9). In this case, even though the base structure is preserved, and the resultant portfolio term structure is sensible and smooth, issue value residuals remain (less than $0.1 \%$ ), and issue structure smoothness is sacrificed as a consequence. Issue structures now contain variance that seemingly primarily serves to attain portfolio forward rates, discount factors, and structure, and that seems hard to justify as unique or informative price information. On the one hand, this may be a consequence of, and response to, market efficiency - the model responding to inefficient pricing. Or, it may simply be that both premises of the relationship between issue and portfolio term structures are not practically simultaneously attainable, requiring a trade-off similar to the goodness-of-fit versus smoothness trade-off of conventional term structure decomposition. 


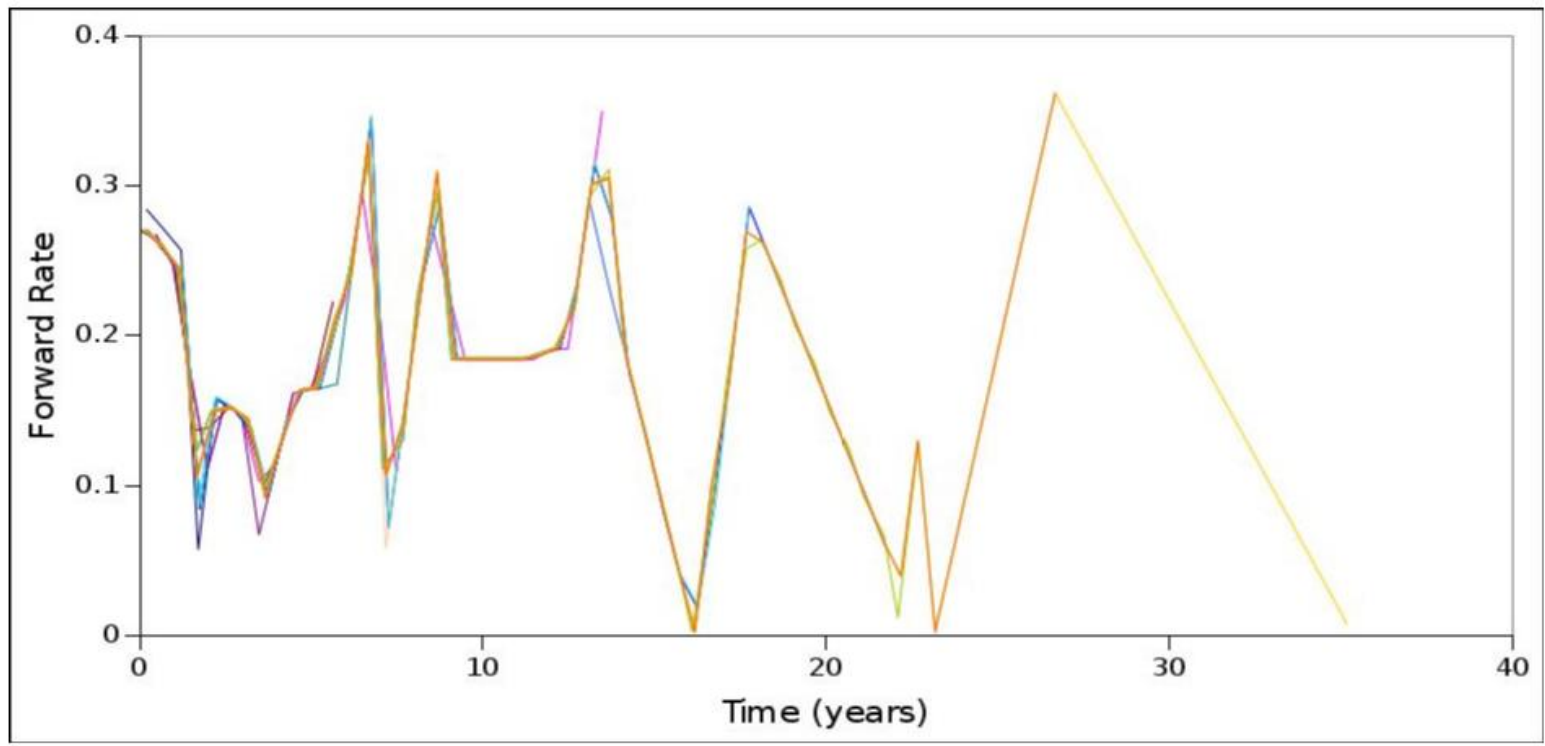

Figure 18. Modelled issue term structures - sequential approach

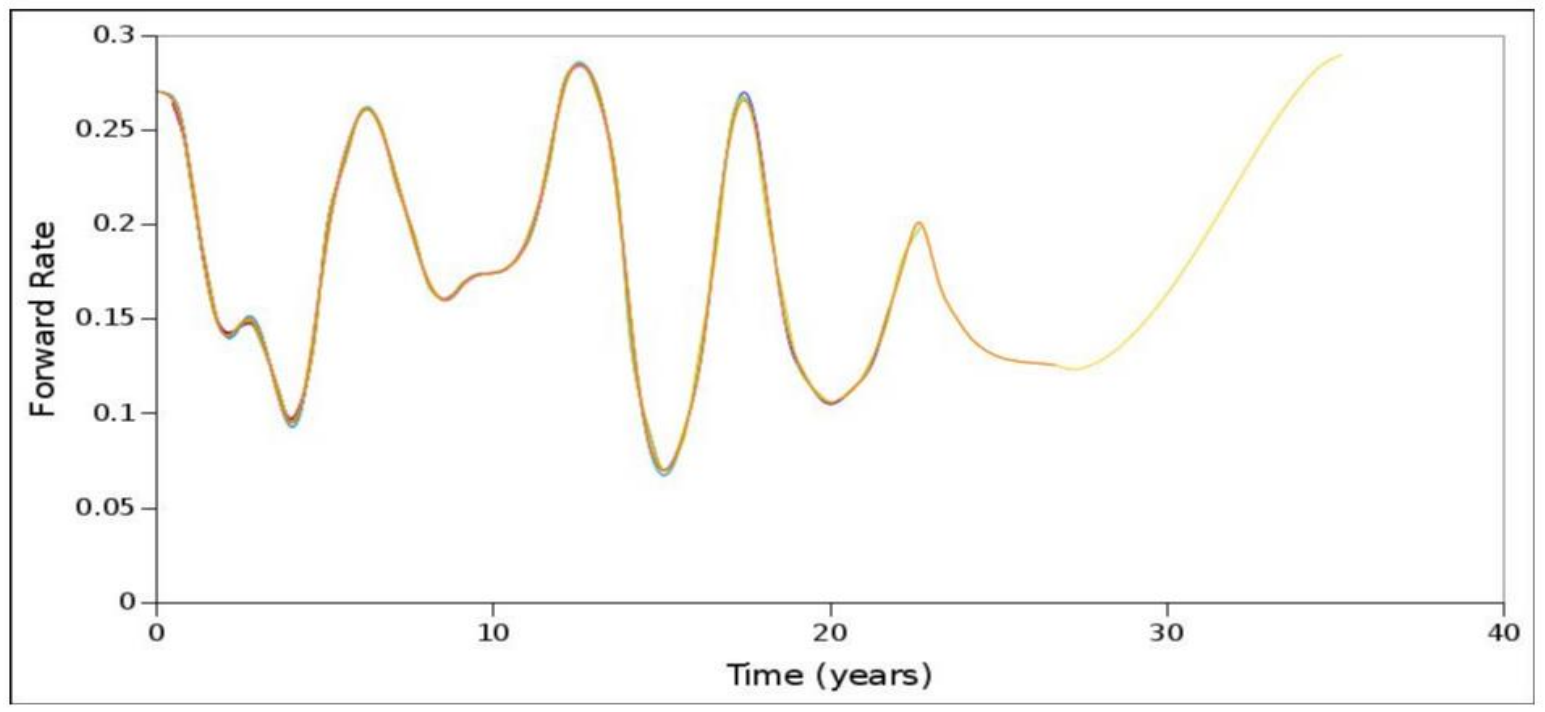

Figure 19. Modelled issue term structures - base structure approach; simulated case

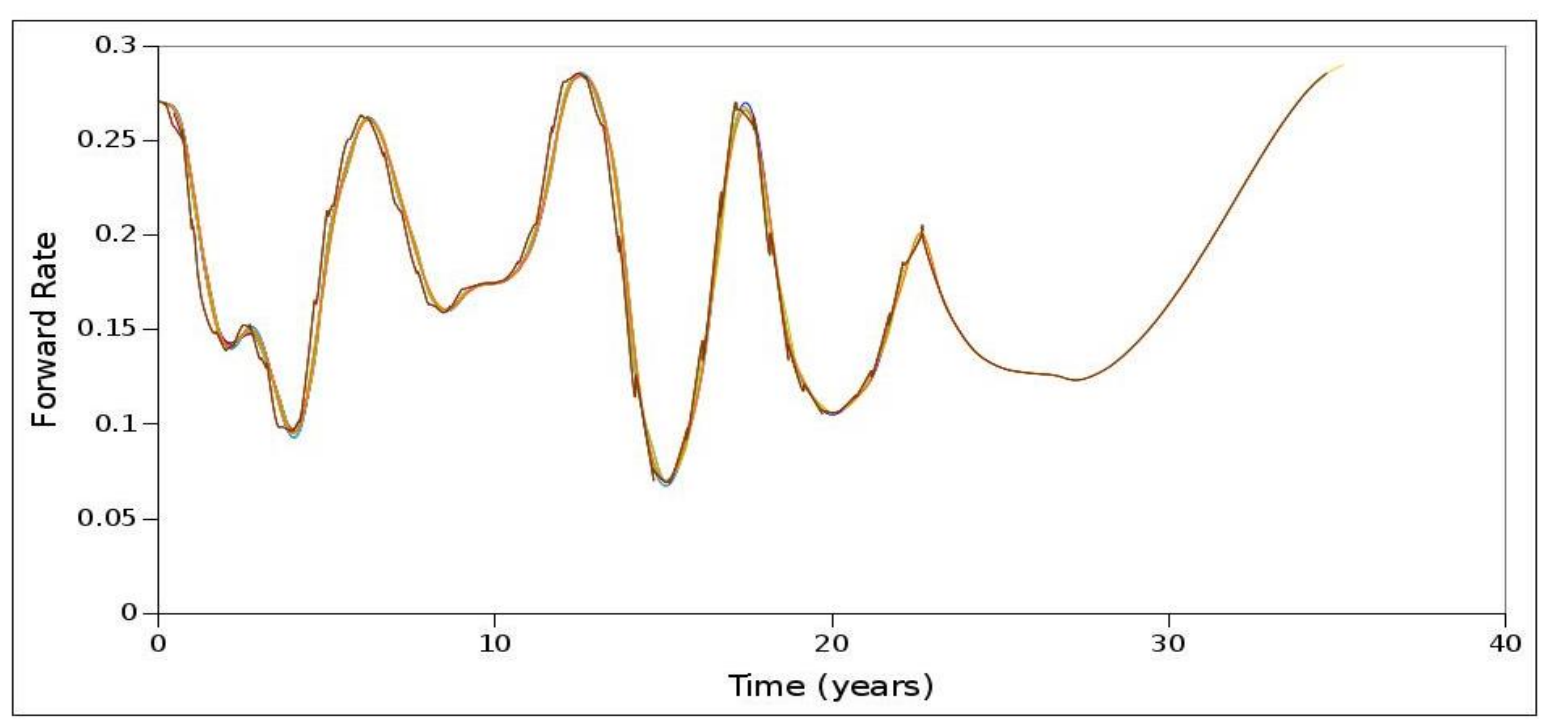

Figure 20. Modelled issue and portfolio term structures - base structure approach; simulated case 


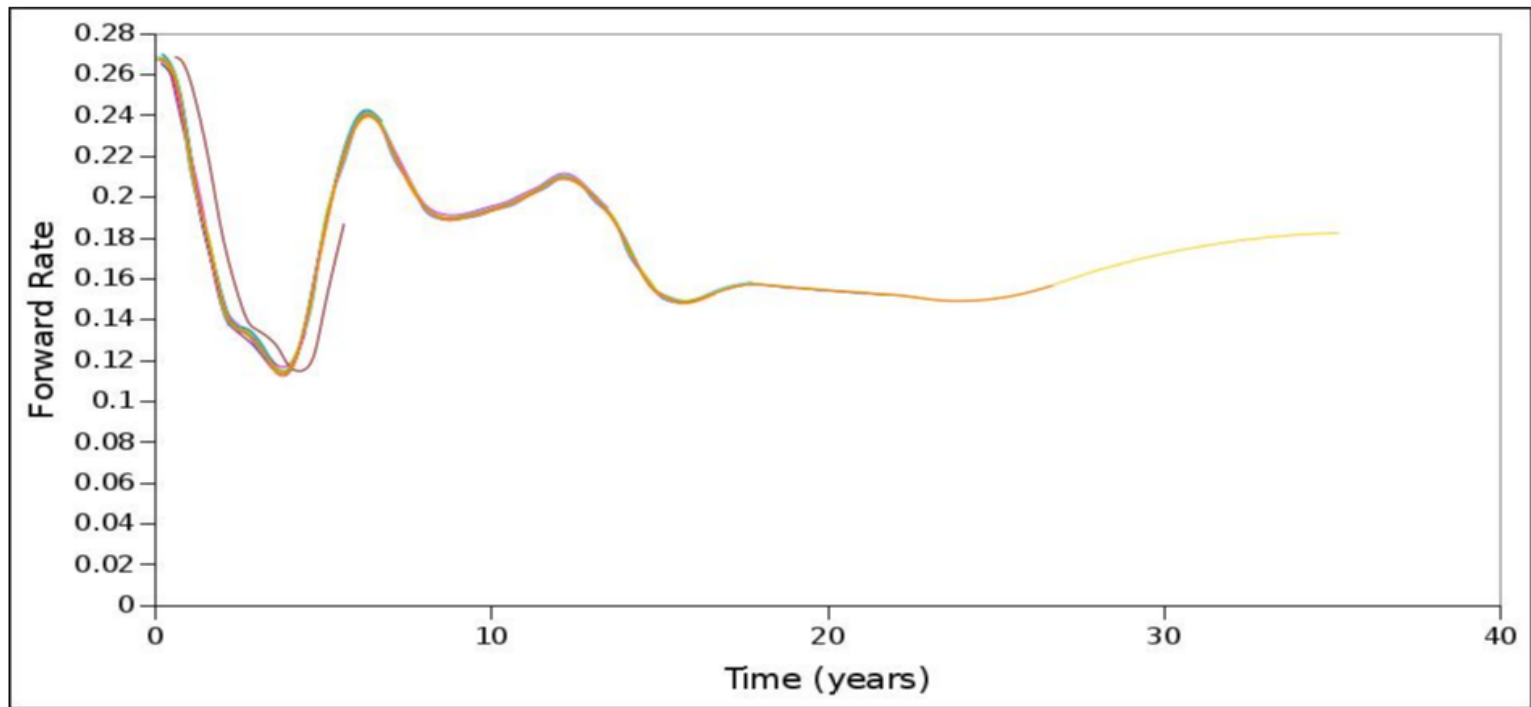

Figure 21. Modelled issue term structures - market case; variance ceiling (0.15)

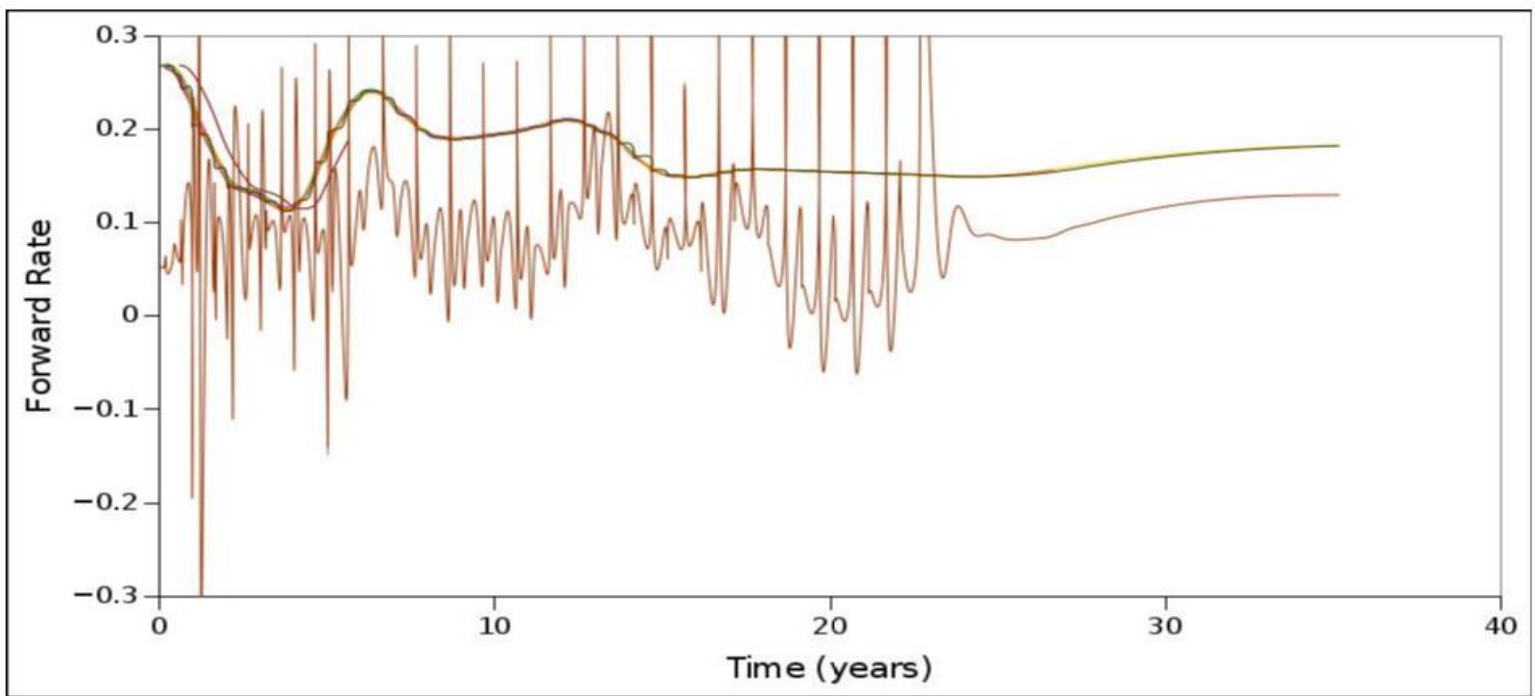

Figure 22. Modelled issue and portfolio term structures - market case; variance ceiling (0.15)

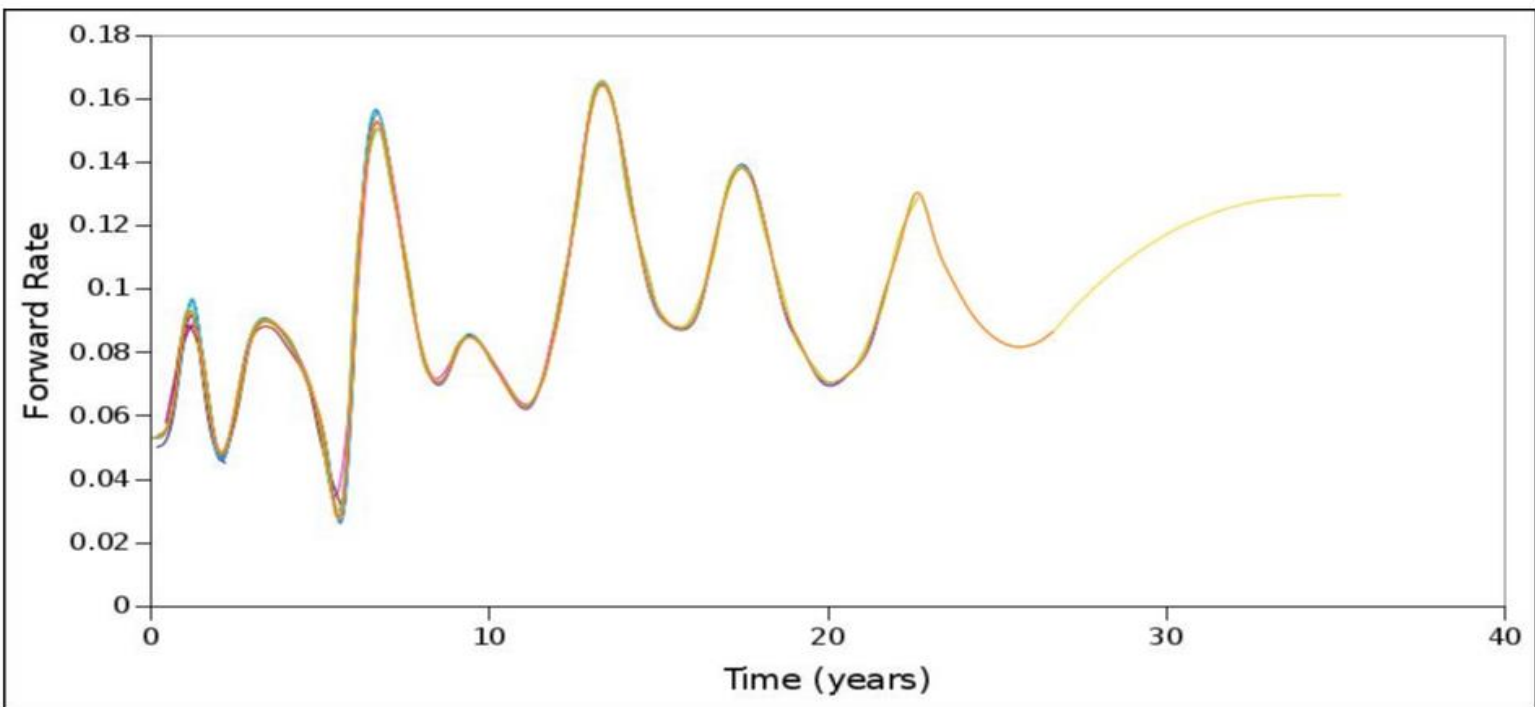

Figure 23. Modelled issue term structures - market case; variance ceiling (0.35) 


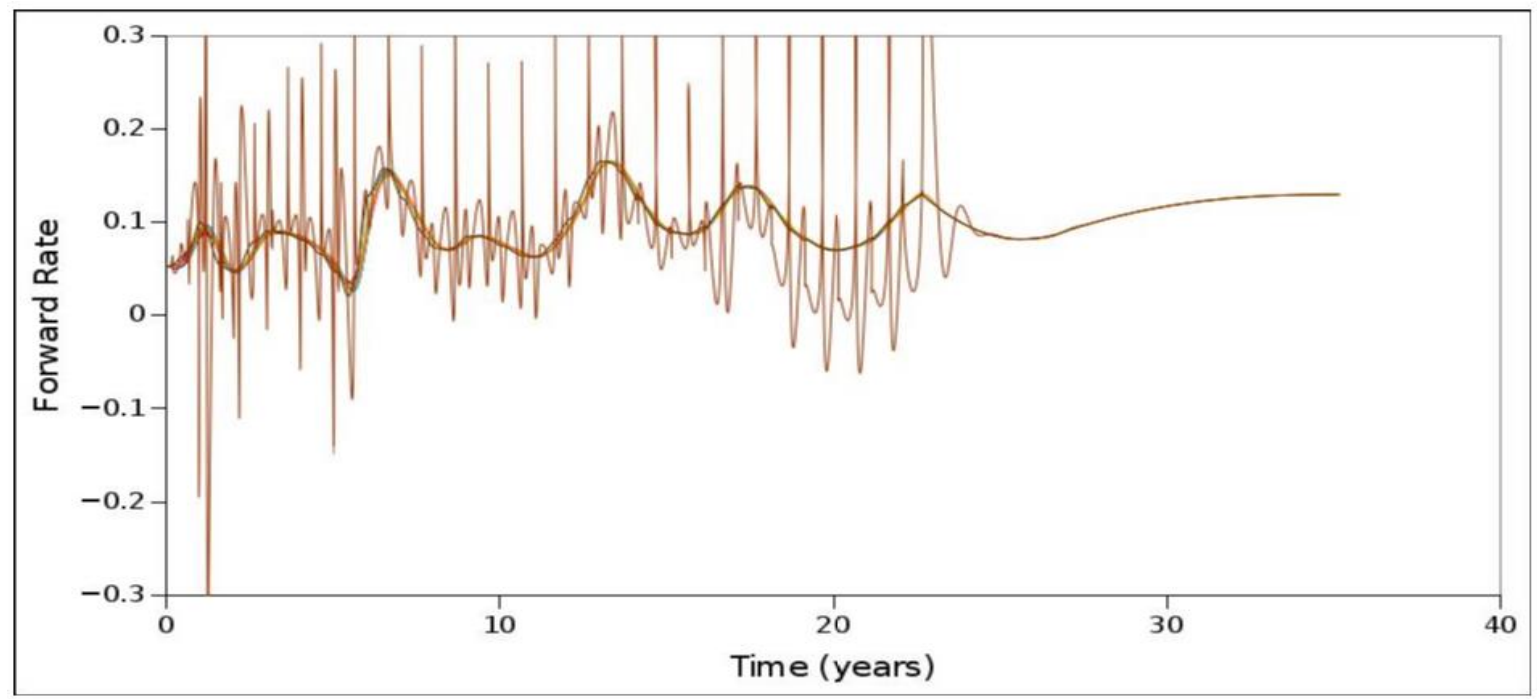

Figure 24. Modelled issue and portfolio term structures - market case; variance ceiling $(0.35)$

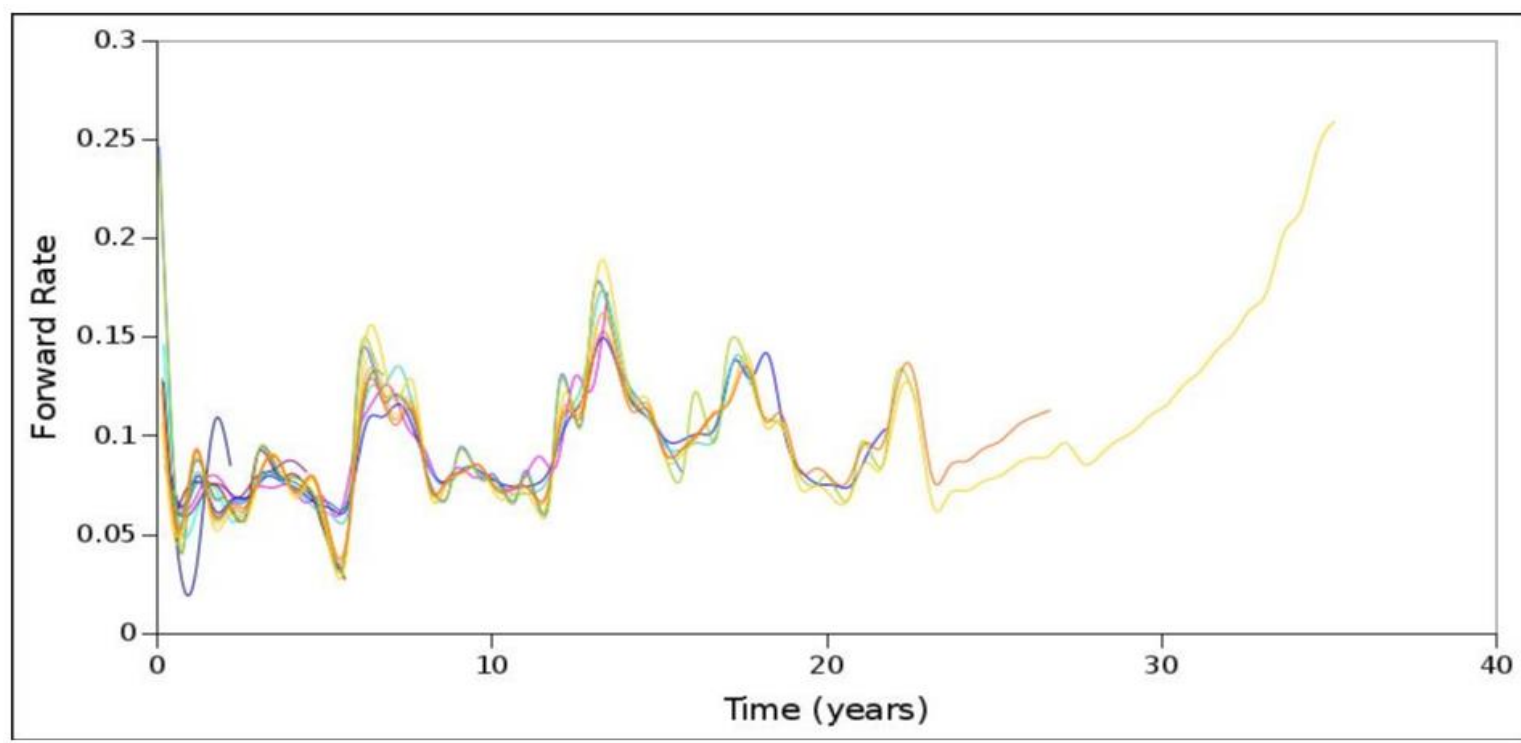

Figure 25. Modelled issue term structures - portfolio variance minimized

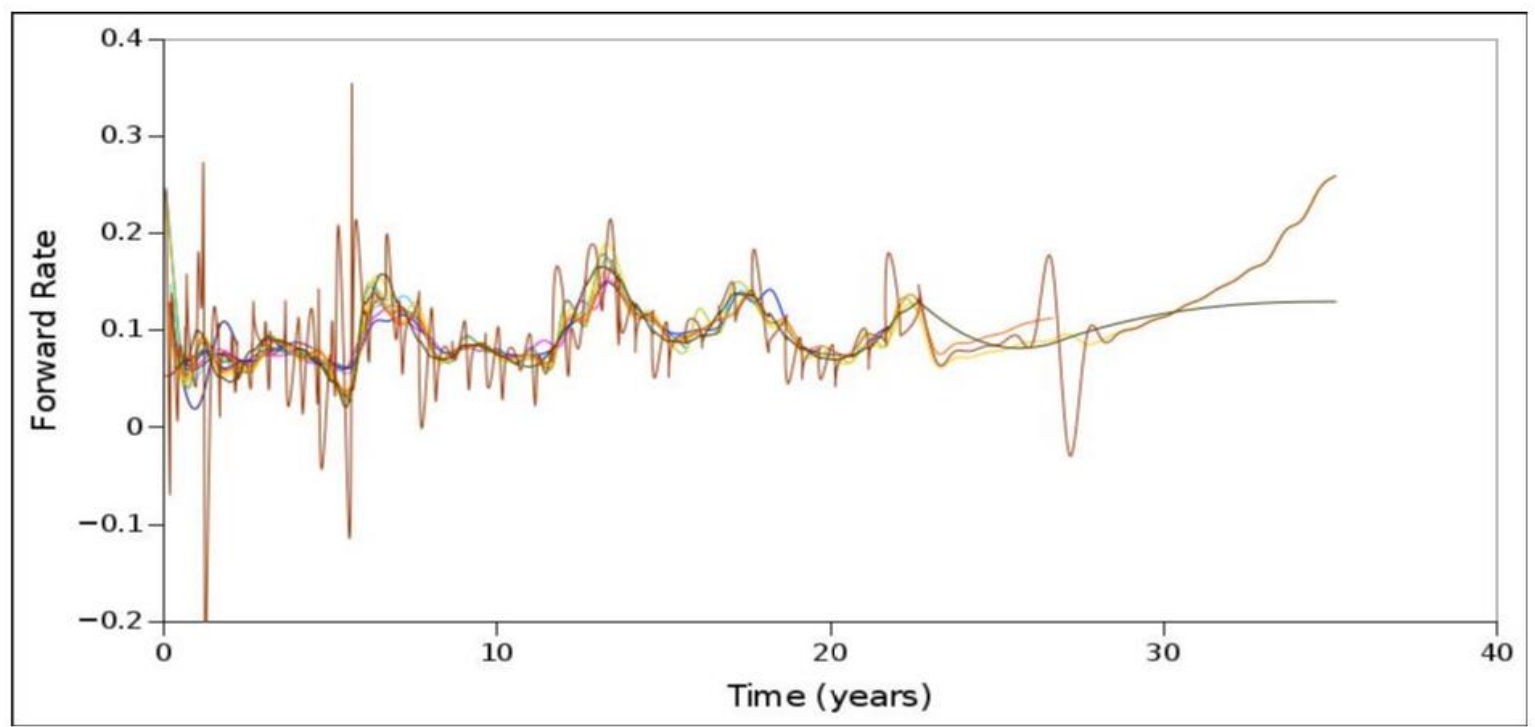

Figure 26. Modelled issue and portfolio term structures - portfolio variance minimized 


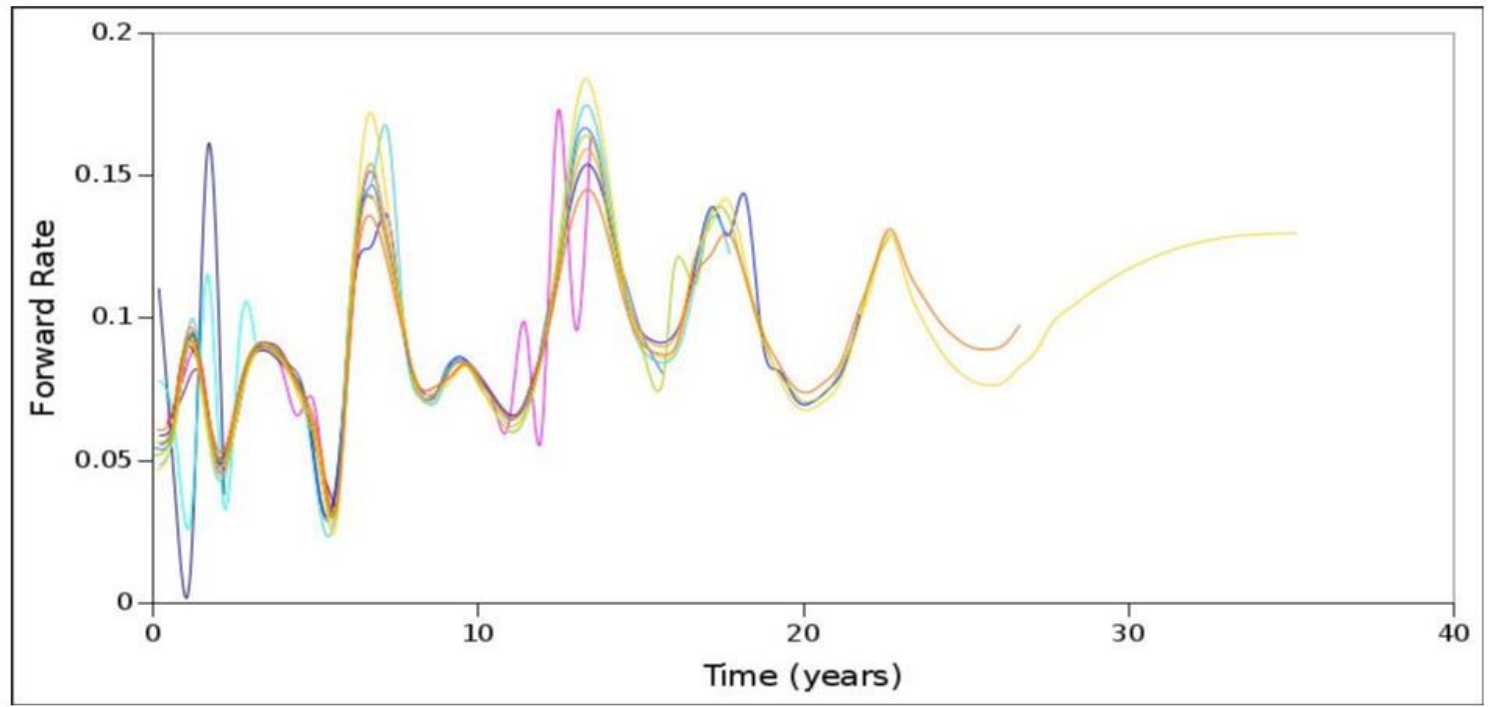

Figure 27. Modelled issue term structures - base structure preserved

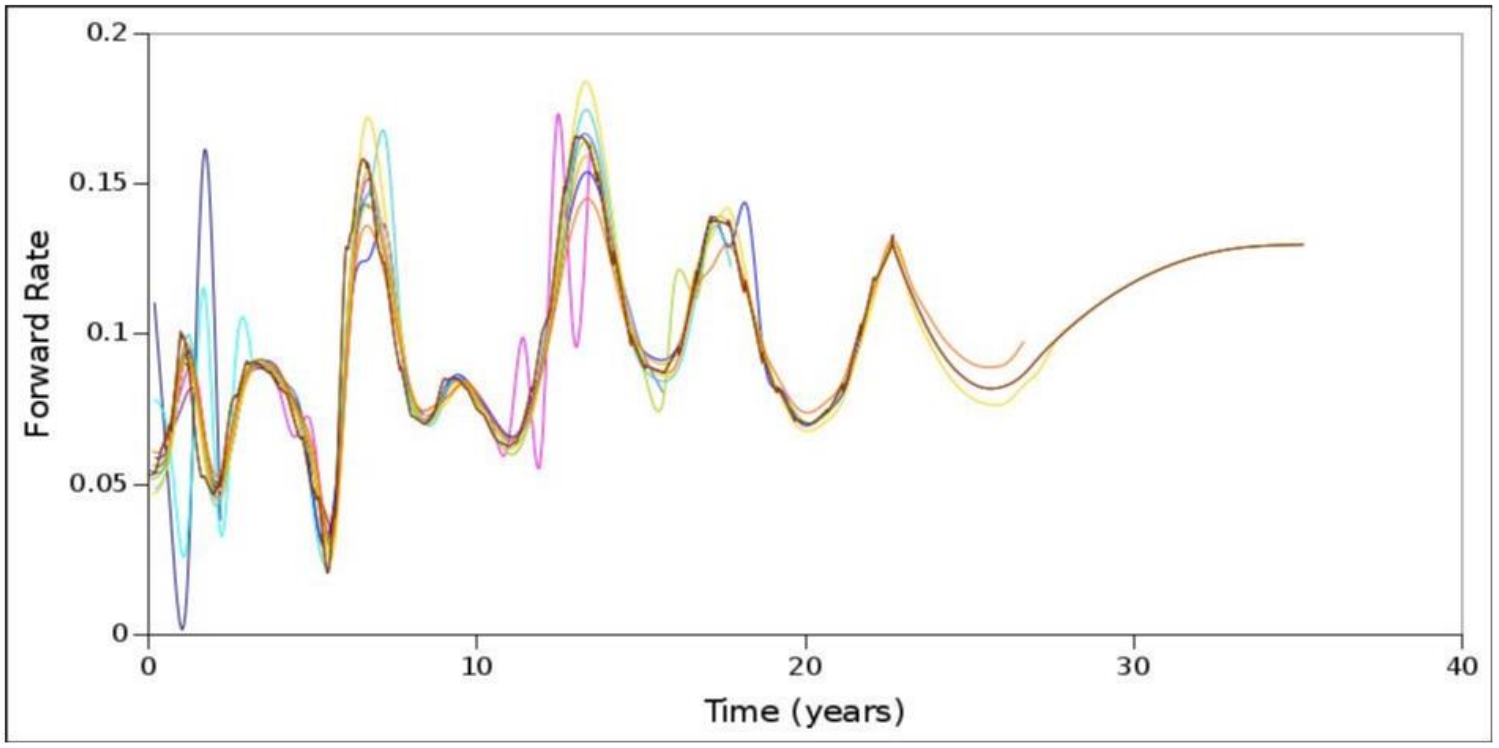

Figure 28. Modelled issue and portfolio term structures - base structure preserved

\section{Conclusion}

Even the worst outcomes make clear the reality of issue term structures, as structures distinct from the portfolio term structure, containing information, just as the portfolio term structure is acknowledged and well known to contain.

The general view why the optimization problems of the axiomatic structural approaches fail to yield proper structure, is that the constraints are not adequate or sufficient to steer the problem across what can only be described as otherwise local optima, due to a too non-linear or poorly defined problem then. As stated under the analysis, minimizing issue value against the derived portfolio term structure as well, may be the missing constraint to secure structure. However, it is questioned whether this can be simultaneously done whilst minimizing issue value against issue term structures - the predominant objective of the axiomatic structural approach optimizations - necessitating two separate steps. It will then also essentially approximate the base structure approach. Both Gauthier and Simonato (2012) and Gimeno and Pineda (2006) note method to overcome highly non-linear decomposition problems, which includes seeding. The base structure approach could then be seen as a seeding method to seed the optimization problems with a base or initial structure.

The base structure approach offers a workable method to decompose at the instrument level, particularly when disregarding the derived portfolio term structure. Two variants of the base structure approach were examined. The method of shifting the base structure to obtain issue term structures offer very smooth issue term structures, even though it does not necessarily yield an appropriate portfolio term structure. The particular approach followed here was to uniformly shift issue forward rates. Attention can perhaps be given to more elaborate means. The second variant of the 
base structure approach preserves portfolio term structure integrity, but sacrifices issue term structure smoothness in favour of portfolio term structure smoothness.

The basic criteria of conventional term structure decomposition - goodness-of-fit, smoothness, and stability - naturally extend to instrument-level term structure decomposition. Also, the trade-off between goodness-of-fit and smoothness may prevail, albeit in different form. It is not altogether certain whether it would be possible to attain both smooth issue term structures, and a smooth portfolio term structure at all times, or even at all. In the end, a trade-off between issue term structure smoothness and portfolio term structure smoothness may practically persist. More complex term structure simulations that consider an unique term structure per issue, or per issue sub-group (assigning issues to sub-groups and simulating term structures for these) should help to answer the question from a theoretical vantage point. Also, the effect of market efficiency should be considered.

The study began to explore the goodness-of-fit properties and smoothness properties of instrument-level term structure decomposition. Subsequent attention can be given to its stability properties, which may be more complex than conventional term structure decomposition, because of the number of instruments or term structures involved.

The pragmatic utility of issue term structures lays in the information they contain. One instance are issues' risk premia explaining why an issue has the risk premium it has. Issue term structures offer more elaborate data to do so. Another, related instance are issues' default probabilities - quantifying issue default probability over the lifetime of the issue. Again, issue term structures offer more elaborate data to do so.

The study examined a risk-free portfolio of bond instruments. Instrument level term structure decomposition should equally apply to risky instruments, and may even find greater application value in such a context, as its intend is to expand and reflect on inherent instrument risk.

\section{References}

Barnard, B. (2019) Interest rate term structure decomposition: an axiomatic structural approach. Applied Economics and Finance, 6(1).

Bolder, D. J., \& Gusba, S. (2002) Exponentials, Polynomials, and Fourier Series: More Yield Curve Modelling at the Bank of Canada, Bank of Canada Working Paper.

Bolder, D. J., Johnson, G., \& Metzler, A. (2004). An empirical analysis of the canadian term structure of zero-coupon interest rates, Bank of Canada Working Paper.

Boyd, S., \& Vandenberghe, L. (2004) Convex Optimization, Cambridge University Press. https://doi.org/10.1017/CBO9780511804441

Elton, E. J., Gruber, M. J., Agrawal, D., \& Mann, C. (2001) Explaining the Rate Spread on Corporate Bonds. The Journal of Finance, 56(1). https://doi.org/10.1111/0022-1082.00324

Gauthier, G., \& Simonato, J. G. (2012) Linearized Nelson-Siegel and Svensson models for the estimation of spot interest rates. European Journal of Operational Research, 219 (2), 442-451. https://doi.org/10.1016/j.ejor.2012.01.004

Gimeno, R., \& Pineda, J. M. N. (2006) Genetic algorithm estimation of interest rate term structure. Documentos de trabajo del Banco de España, (34), 9-36.

Li, B., DeWetering, E., Lucas, G., Brenner, R., \& Shapiro, A. (2001) Merrill Lynch Exponential Spline Model, Merrill Lynch Working Paper.

Marciniak, M. (2006) Yield curve estimation at the National Bank of Poland. Bank i Kredyt, 10, 52-74.

\section{Copyrights}

Copyright for this article is retained by the author(s), with first publication rights granted to the journal.

This is an open-access article distributed under the terms and conditions of the Creative Commons Attribution license which permits unrestricted use, distribution, and reproduction in any medium, provided the original work is properly cited 